Review Article

\title{
Research Progress Review of Preparation and Applications of Fluorescent Hydrogels
}

\author{
Weiwei Su, ${ }^{1}$ Ran Wang, ${ }^{1}$ Cheng Qian, ${ }^{2,3}$ Xuetong Li, ${ }^{3}$ Qi Tong, ${ }^{1}$ and Tifeng Jiao ${ }^{1}{ }^{1}$ \\ ${ }^{1}$ Hebei Key Laboratory of Applied Chemistry, \\ Heavy Metal Deep-Remediation in Water and Resource Reuse Key Lab of Hebei Province, \\ School of Environmental and Chemical Engineering, Yanshan University, Qinhuangdao 066004, China \\ ${ }^{2}$ School of Electrical Engineering, Yanshan University, Qinhuangdao 066004, China \\ ${ }^{3}$ National Engineering Research Center for Equipment and Technology of Cold Strip Rolling, Qinhuangdao 066004, China
}

Correspondence should be addressed to Tifeng Jiao; tfjiao@ysu.edu.cn

Received 16 July 2020; Revised 21 October 2020; Accepted 11 November 2020; Published 29 November 2020

Academic Editor: Jo o Paulo Leal

Copyright (c) 2020 Weiwei Su et al. This is an open access article distributed under the Creative Commons Attribution License, which permits unrestricted use, distribution, and reproduction in any medium, provided the original work is properly cited.

The fluorescent gel with good flexibility and biocompatibility has attracted more and more attention due to its excellent optical properties. In this paper, the research progresses in preparation methods and applications of fluorescent gels are reviewed. In addition, the preparation methods of self-assembly and polymerization of fluorescent gel are also introduced. In this paper, it should be noted that some outstanding research about the fluorescent gels used in sensors, bio-imaging probes, drug delivery, and other application fields is summarized. This work provides useful reference information for further exploration and study of fluorescent hydrogels.

\section{Introduction}

In recent years, the development of hydrogels has attracted the attention of researchers. Hydrogel is a polymer with a three-dimensional network structure swollen by water [1]. Hydrogels can be classified into two types according to their sources: synthetic and natural hydrogels. Natural hydrogels have higher water absorption capacity, and their hydrophilic structures enable them to contain large amounts of water in their threedimensional network. In addition, natural hydrogels show good flexibility, biocompatibility, and long service life [2]. Moreover, due to its similar structure to extracellular matrix, natural hydrogel can also be used in tissue engineering fields such as drug delivery and cell transfer. Synthetic hydrogel is rich in raw materials and has a long service life. Compared with natural hydrogels, synthetic hydrogels have better mechanical strength and stability and wider adjustable range of structure and performance, which make them play an important role in biomedical, biosensing, and tissue engineering fields.
Fluorescence is a common phenomenon, which has been studied in detail all over the world. In 1970, Galley et al. independently found that the spectral properties of aromatic fluorophores embedded in different rigid and highly viscous media did not conform to the classical rules [3]. The fluorescence spectrum shows different color fluorescence, which also depends on the excitation wavelength; different excitation leads to different color states. The distribution of excited states and the different dielectric relaxation rate in a certain environment promote the different emission of fluorescence.

Fluorescent hydrogel is a kind of polymer gel, which has attracted the attention of scientists because of its special luminescent properties [4-8]. Fluorescent gel can be regarded as the combination of light and gel, which possesses excellent properties of hydrogel. Adjusting the chromophore and the environment of the excited state can change the state of the fluorescent hydrogel and the change of its emission spectrum, making it have great significance in the fields of biosensor, fluorescence probe, imaging tracking, and cancer treatment. This review mainly introduces the 
preparation methods, types, and properties of fluorescent hydrogels, as well as their application in related fields, and looks forward to their application research and development prospects.

\section{Preparation Method}

With the expansion of the application field of hydrogels, people have more requirements for their performance. In order to improve the comprehensive performance of fluorescent hydrogels and better meet the requirements of different application fields, researchers have developed different preparation methods. The water-soluble or hydrophilic polymers can form hydrogels through certain chemical or physical crosslinking. The fluorescent hydrogels can be prepared by self-assembly, polymerization, crosslinking, and other methods. Among them, self-assembly belongs to physical crosslinking and it is a method to prepare hydrogels based on weak interaction forces. Polymerization is a kind of chemical crosslinking, because chemical reaction occurs, which causes the intermolecular covalent bond to act to form hydrogel. From these two parts, the preparation method of fluorescent hydrogel is briefly described below.

2.1. Physical Crosslinking. Physical crosslinking is the formation of hydrogels through physical forces such as electrostatic interaction, ion interaction, hydrogen bonding, and chain winding. At present, self-assembly is the most common physical crosslinking method, and self-assembly plays an important role in various technical fields $[9,10]$. Selfassembly is a process in which small structural units are spontaneously arranged to form an ordered structure without human intervention, which exists in natural and unnatural systems. These structural units consist of molecules, nano- and micromaterials, or larger materials. Based on the interaction of noncovalent bonds (such as hydrogen bond, van der Waals force, electrostatic interactions, and $\pi-\pi$ interactions), they also spontaneously organize into a series of regular structures. The following is a brief description of the preparation of hydrogels from these different weak interaction forces.

2.1.1. Electrostatic Interaction. The fluorescent hydrogel can be prepared by electrostatic interaction. For example, Xia et al. [11] reported a self-assembled peptide based printable fluorescent hydrogel, which has the ability to grow the beta folded fiber through self-assembly [12-14]. Here, the sequence of the ion complementary peptide is selected as the self-assembly motif, and the $\mathrm{N}$-terminal of the peptide is capped by the powerful ligand 2,2-bipyridine (Bpy), for metal ion chelation (Figure 1(a)). According to the investigation, the metal ion $\mathrm{Eu}^{3+}$ has excellent fluorescence performance, so it is selected as the metal ion center here. The self-assembled motif contains lysine $(\mathrm{K})$ of the side chain protonated amino group and glutamic acid (E) with carboxylic acid on the side chain. The electrostatic interaction between positive and negative ions, coupled with the hydrophobic interaction between phenylalanine $(\mathrm{F})$ residues and the pion stacking effect, can be self-assembled into a fibrous hydrogel structure under neutral pH (Figure 1(b)). Bpy-KFEFKFEF with the combination of metal ions $\mathrm{Eu}^{3+}$, $\mathrm{Eu}^{3+} \mathrm{Eu}^{3+}, \mathrm{Eu}^{3+}$ and Bpy". Please check and confirm the correct usage." and Bpy, and the hydrogel has photoluminescence properties (Figures 1(c) and 1(d)).

2.1.2. Hydrogen Bonding. In solution systems, intramolecular and intermolecular hydrogen bonding can also act as a physical crosslinking point. Chu et al. [15] selected two nonaromatic functional amino acids, serine (S/Ser) and aspartic acid (D/Asp), and used them as side chains of amino acids (Figure 2(a)). Their unique structure can generate extended hydrogen bond networks during self-assembly. There are different side chains of amino acids, and the $\mathrm{pH}$ value of self-assembly is also different, which is $\mathrm{pH}$ dependent. The hydroxyl containing tetraphenylethene-serine acid (TPE-Ser) with the lowest molecular weight will selfassemble into supramolecular hydrogels under physiological $\mathrm{pH}$ conditions. Tetraphenylethene-aspartic acid (TPE-Asp) side chains with carboxyl groups will undergo self-assembly and hydrogelling process under weak acidic conditions (Figure 2(b)). Because TPE has strong hydrophobicity, the rotation of phenyl group is limited and the molecules of benzene ring cannot rotate in aqueous medium, which makes the space limited luminescence, so the gel shows enhanced fluorescence intensity. Yan et al. [16] reported a reversible multi-sensitive quantum dot gel formed by hyperbranched polyamidoamine (PAA) on the surface of functional fluorescent semiconductor quantum dots (QDs). Furthermore, the formed QDs gel showed stronger fluorescence than the corresponding solution.

2.1.3. Other Weak Interaction Forces. There are many reports about the physical crosslinking of hydrogels [17]. In addition to electrostatic interaction and hydrogen bonding, weak interactions also exist in the gels, such as the $\pi-\pi$ stacking and hydrophobic interactions. For example, Ji et al. [18] have successfully synthesized luminescent supramolecular crosslinked polymer gel based on benzo-21-crown-7 (B21C7)/dialkyl ammonium salt host-guest interaction with TPE. Because TPE has the effect of aggregation induced emission (AIE), the luminescence intensity of hydrogel will also increase with the increase of concentration. Zhang et al. [19] also applied the crosslinking method. A supramolecular polymer gel with AIE effect was prepared by the combination of host-guest interaction and TPE. Subject-guest interaction is also a weak interaction, including $\pi-\pi$ stacking and hydrophobic interaction. In addition, non-coordination action is also a kind of weak interaction force. Bhowmik et al. [20] proposed a new design strategy of rare Earth luminescent materials based on self-assembly, prepared a supramolecular gel doped with lanthanide complexes, and sensitized the gel by doping pyrene into the gel in a noncoordinated manner. Lanthanide ions are part of the gel matrix and participate in the self-assembly process. At the same time, hydrogel as a matrix of lanthanide enhances the luminescence potential. 


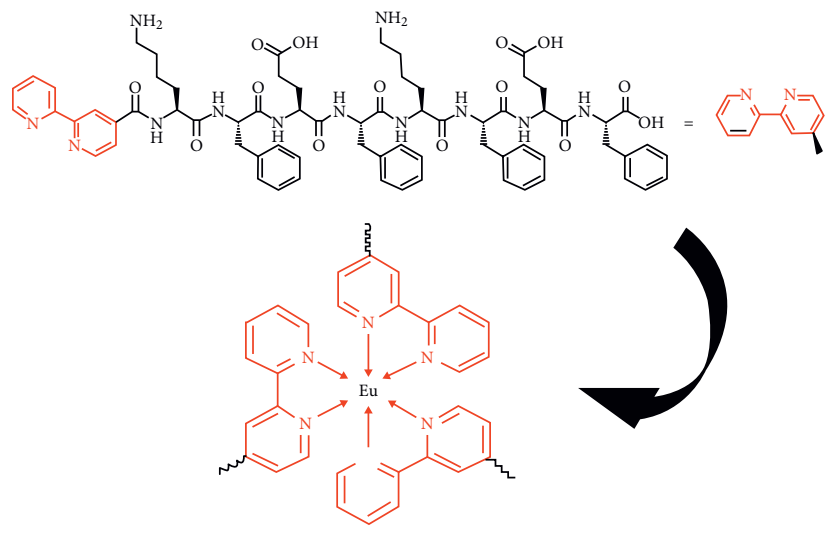

(a)

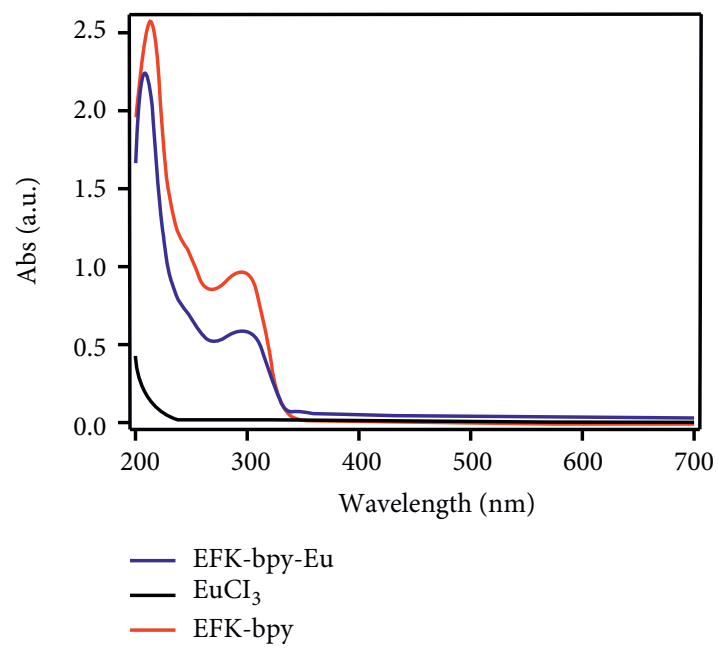

(c)

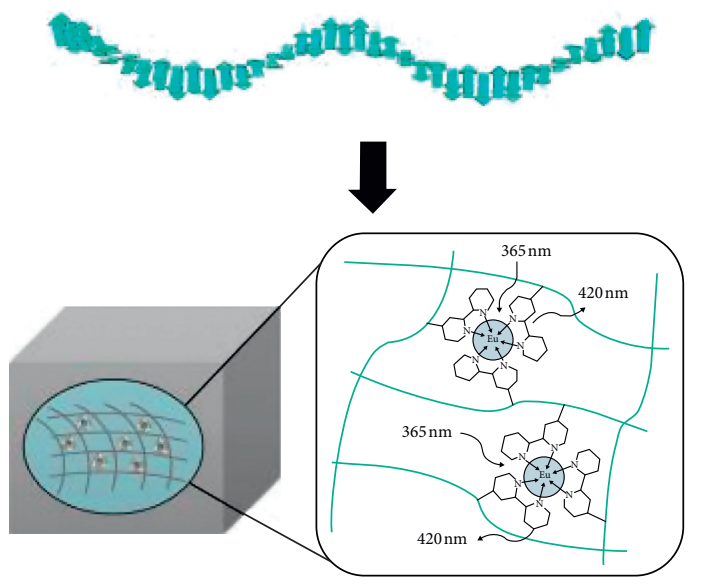

(b)

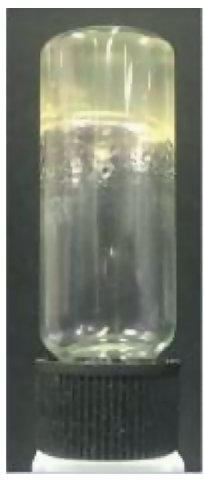

Natural

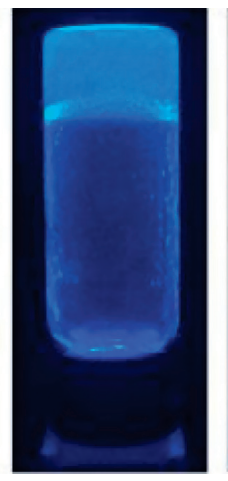

UV1

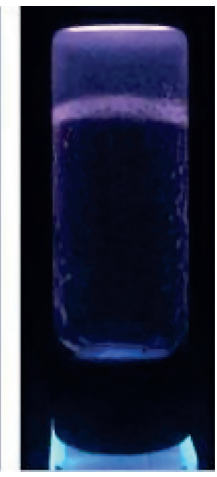

UV2 (d)

Figure 1: Schematic diagram and related fluorescence diagram of self-assembled peptide. (a) The molecular structure of EFK-Bpy peptide and the structure of EFK-Bpy-Eu peptide. (b) Schematic diagram of co-assembled hydrogel and energy transfer process. (c) UV-Vis absorption spectra of EFK-Bpy hydrogel $(4 \mathrm{mM}), \mathrm{EuCl}_{3}(1.3 \mathrm{mM})$, and EFK-Bpy-Eu hydrogel (1.3 mM). (d) Optical images of EFK-Bpy-Eu hydrogels irradiated by natural light (left), ultraviolet $1(330-390 \mathrm{~nm})$ and ultraviolet $2(220-280 \mathrm{~nm})$ (reprinted from [11]).

2.2. Chemical Crosslinking. Chemical crosslinking refers to the connection of polymer chains through chemical reaction. Generally speaking, when external force is applied, the fluidity of crosslinked gel will decrease. In addition, the swelling property will decrease, and the chemical resistance and solvent resistance will increase [21]. Chemical hydrogels are three-dimensional network polymers formed by chemical bonds and crosslinking, which are irreversible. Among them, different crosslinking agents also have different effects on the formation of hydrogels. By changing the concentration and properties of the crosslinking agent, the external environment at the time of crosslinking, such as temperature, can precisely adjust the mechanical properties in the linear and strain strengthened state [22]. Polymerization is one of the chemical crosslinking methods and plays an important role in the preparation of hydrogels. It is a common form in the formation of fluorescent hydrogels, including free radical polymerization, precipitation polymerization, emulsion polymerization, and in situ polymerization.

2.2.1. Emulsion Polymerization. Emulsion polymerization usually refers to the polymerization reaction initiated by heat, light, radiation, and initiator in the microemulsion system with the polymerizable monomer as the dispersed phase and water or other media as the continuous phase. In 2010, Shibata et al. [23] prepared injectable hydrogel beads by polymerization. They first synthesized the fluorescent monomer GF consisting of glucose recognition sites, fluorescent sites, spacers, and polymerization sites. Subsequently, the fluorescent monomer GF was polymerized with acrylamide monomer and crosslinking agent, and then the fluorescent polyacrylamide hydrogel beads with injectable size were prepared (Figure 3). Because of the good hydrophilicity and biocompatibility of acrylamide, the use of 
<smiles>O=C(N[C@@H](CO)C(=O)O)c1ccc(C(=C(c2ccccc2)c2ccccc2)c2ccccc2)cc1</smiles>

TPE-Ser<smiles>O=C(O)C[C@H](NC(=O)c1ccc(C(=C(c2ccccc2)c2ccccc2)c2ccccc2)cc1)C(=O)O</smiles>

TPE-Asp

(a)

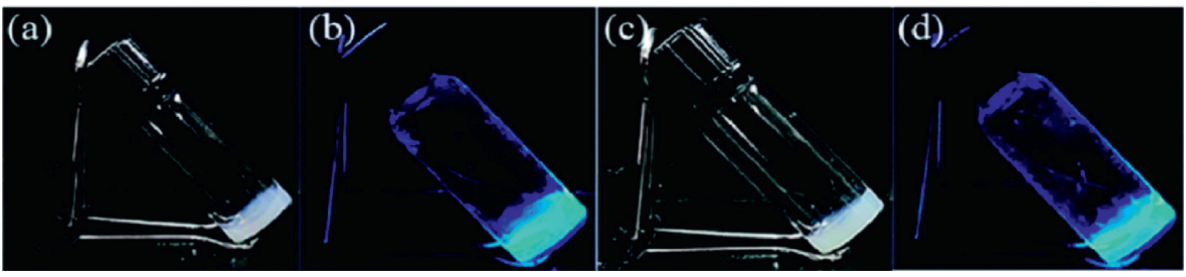

(b)

Figure 2: The structural formula of TPE-Ser and TPE-ASP and the optical picture after self-assembly. (a) The structural formula of TPE-Ser and TPE-ASP, (b) TPE-Ser $(2 \% \mathrm{w} / \mathrm{v}, \mathrm{pH}=7.1)$ and TPE-Asp $(2 \% \mathrm{w} / \mathrm{v}, \mathrm{pH}=6.0)$ self-assembling optical images of hydrogels. Among them, the first two were TPE-Ser gels, and the other two were TPE-Asp gels. (A) (C) are ordinary light, and (B) (D) are ultraviolet light (reprinted from [15]).

$$
\mathrm{H}_{2} \mathrm{~N}^{\prime \text { PEG }} \prod_{\mathrm{O}}^{\mathrm{OH}}
$$
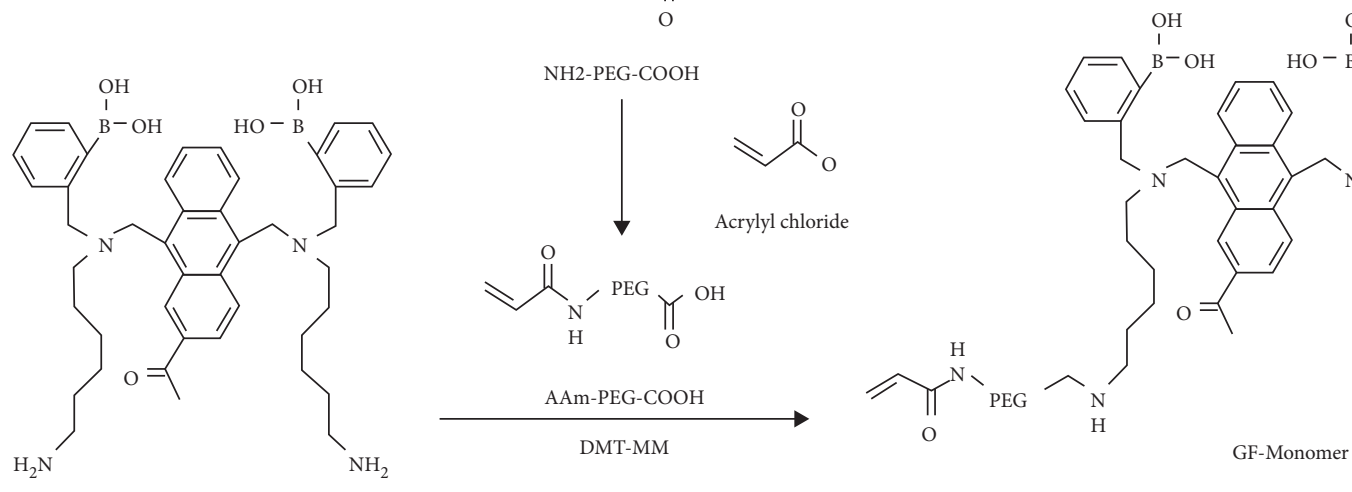

GF-Monomer
$\mathrm{OH}$

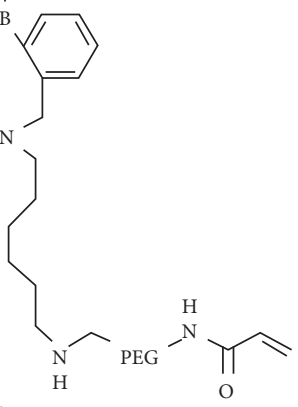

GF-dye

(a)

Figure 3: Continued. 


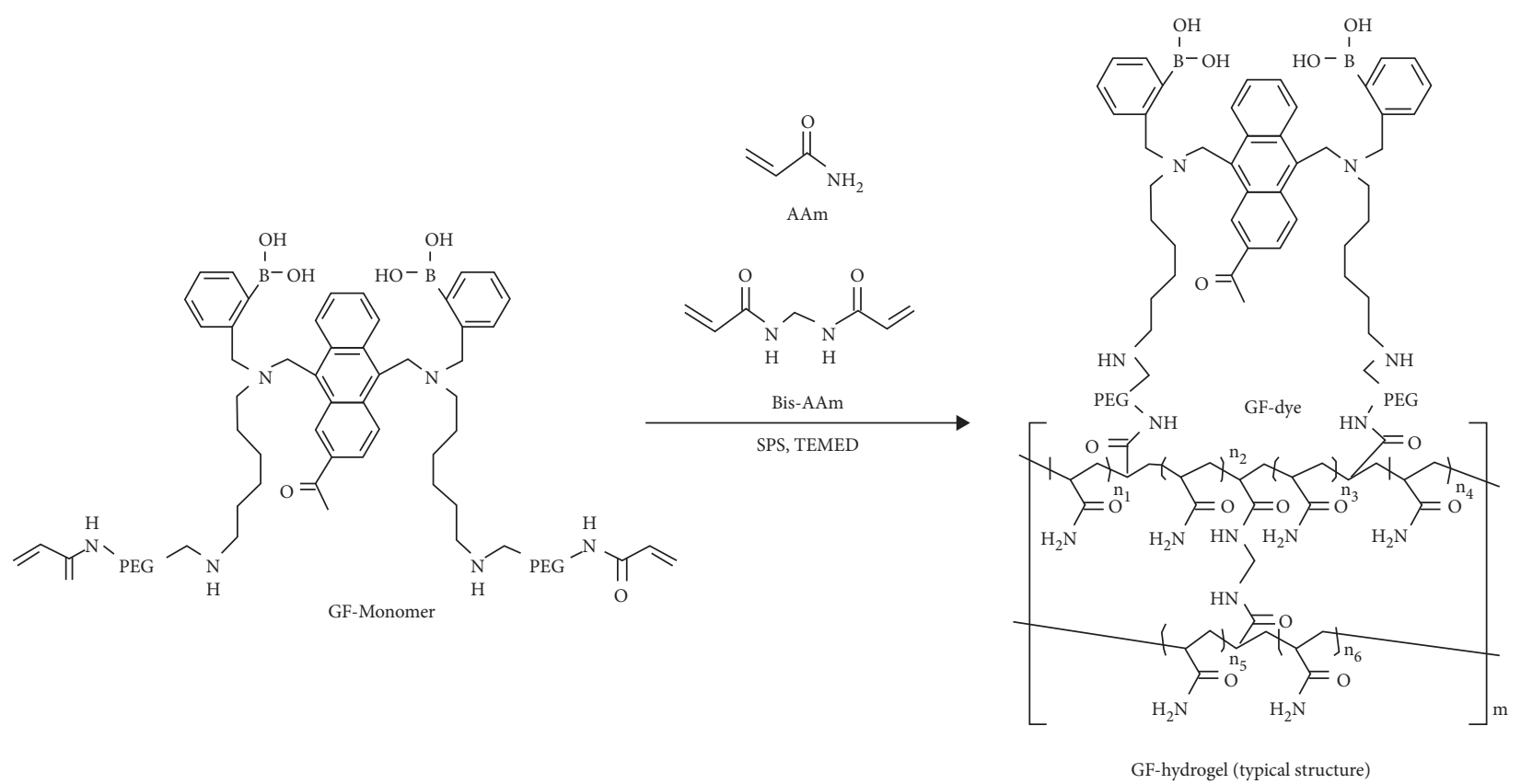

(b)

FIGURE 3: Synthesis of fluorescent monomer (GF-monomer) and hydrogel (GF-hydrogel). (a) Preparation of GF monomer. (b) Preparation of GF hydrogel (reprinted from [23]).

acrylamide as a polymerization site can greatly promote the polymerization process.

In addition, numerous studies have been conducted on fluorescent hydrogels, which have been used to synthesize various functional fluorescent gels. Nishiyama et al. [24] synthesized polyacrylic acid (PAAc) gel particles containing DNA by emulsion polymerization, and certain chemical reagents were added to assist in the polymerization process, for example, initiator potassium persulfate, crosslinking agent $n, n$-methylene bisacrylamide, etc. Then, PAAc gel particles containing DNA were immersed in bromo-ethane (EtBr) solution to prepare PAAc gel particles with DNA$\mathrm{EtBr}$ complex. The fluorescence intensity of PAAc gel particles embedded in DNA-EtBr complex showed high sensitivity and was found to decrease exponentially with $\mathrm{pH}$. Therefore, it can be used in the development of optical micro $\mathrm{pH}$ sensor.

2.2.2. Free Radical Polymerization. Free radical polymerization can be finished by a water-soluble molecule which contains a double bond. The crosslinker is usually a molecule containing at least two polymerizable double bonds, which can generate free radicals during the reaction and then conduct polymerization.

Chen et al. [25] have prepared an easily synthesized thermal responsive organic hydrogel and used covalent bonded 10-phenophenthiazine (PTH) to achieve controlled free radical polymerization. Various monomers (acrylate, methacrylate, acrylamide, vinyl ester, and vinyl amide) were polymerized by reversible addition-fragmentation chain transfer polymerization (RAFT) and atom transfer radical polymerization (ATRP). The study reported that the "high and low" temperature of the fluorescent hydrogel, the "switch" of the light, and the "in and out" of the catalyst could switch the real-time controlled radical polymerization reaction. The "and" gating of controlled radical polymerization is realized, which provides a valuable idea for the design of logic control.

2.2.3. Precipitation Polymerization. Precipitation polymerization is a process in which the resulting polymer does not dissolve in monomers but is precipitated continuously as the reaction progresses. The monomer and initiator can be dissolved in the reaction medium, and the polymer is insoluble in the reaction medium during the reaction. Wang et al. [26] prepared a hybrid nano-hydrogel, which was prepared by precipitation polymerization of polyethylene glycol (PEG) macromonomer, chitosan, and graphite carbon dots (CDs) in aqueous medium in one pot without surfactant. Chitosan chain and CDs are crosslinking agents in aqueous solution, so that hydroxyl and amino groups on the repeating unit of chitosan chain, hydroxyl and carboxyl groups on CDs surface, and the ether oxygen on the PEG macromolecule monomer can form hydrogen bond association or complexation. Then, PEG and other macromolecules were used as monomers for one-pot precipitation polymerization. When these PEG macromonomers are polymerized and crosslinked, chitosan chains will be physically entangled in the formed nonlinear PEG chain network, and CDs will be fixed in situ in the polymerized gel network, where the fixation of CDs is due to hydrogen bonding. When these aqueous dispersions of hybrid nanogels are exposed to ultraviolet rays $(365 \mathrm{~nm})$, they will obviously emit green-blue light. Due to the embedding of 
CDs, the hybrid nano-fluorescent hydrogel also retains the characteristics of CDs and has $\mathrm{pH}$ responsiveness and highefficiency synergistic response, which makes it have great potential in medical diagnosis and treatment in vivo.

2.2.4. In Situ Polymerization. In situ polymerization is developed from nanocomposites and is a method to disperse nanoparticles and polymerize them in situ under certain conditions. Zhu et al. [27] synthesized the thermal sensitive poly (N-isopropylacrylamide)/Au nanocomposite fluorescent hydrogels (PNIPAM/Au) by in situ gamma irradiation assisted polymerization, which initiated the polymerization in situ by inducing the reduction of aqueous chloroauric acid $\left(\mathrm{HAuCl}_{4} \cdot \mathrm{H}_{2} \mathrm{O}\right)$. These studies keep emerging, providing a broader space for the development of fluorescent gel in the future.

\subsubsection{Other Chemical Crosslinking Methods. All fluorescent} gels prepared by chemical reaction belong to chemical crosslinking method. In addition to the above chemical crosslinking methods, there are other crosslinking methods such as graft copolymerization and complexation crosslinking. For example, Kawa and Takahagi [28] made terbium (III) nuclear dendrimer copolymers with n-isopropylacrylamide and prepared a transparent hydrogel which can produce green fluorescence. Ma et al. [29] reported a method of using glutaraldehyde crosslinked bovine serum albumin (BSA) (or human serum albumin (HSA)) to form a new luminescent biological hydrogel (Figure 4(a)). Glutaraldehyde (GA) has low cost, dry reactivity, and good chemical stability compared with the same kind of aldehydes, so it is widely used as a good crosslinking agent [30]. The crosslinking of glutaraldehyde and BSA mainly occurs on the $\alpha$-amino group of lysine [31]. In this study, a high concentration of BSA solution was used to generate a large number of $3 \mathrm{D}$ networks during the intermolecular crosslinking process, thus forming a gel phase, and the gel showed strong green and red autofluorescence in the larger excitation wavelength range (Figure $4(\mathrm{~b})$ ). The results of UV-Vis and fluorescence spectra indicate that three kinds of fluorescent compounds may form in BSA hydrogels with GA as crosslinking agent. The luminescence of this gel is speculated, which may be attributed to four aspects: firstly, secondary amines and ethylenediamines in the molecule are believed to contribute to the fluorescence mechanism. Second is electronic transition, such as $\pi-\pi^{*}$ transition of $\mathrm{C}=\mathrm{C}$ bond. Third is synergism of several amino acids close to each other. Lastly, the charge around the fluorophore in BSA hydrogel is another potential factor affecting fluorescence. In short, a new fluorescent gel was prepared by crosslinking method, and the mechanism of its luminescence could be further studied and discussed. In addition to GA, genipin is commonly used as crosslinking agent. As a water-soluble bifunctional crosslinking agent, genipin is highly respected in the preparation of crosslinked fluorescent gel. For example, Muzzarelli [32] used genipin to rapidly crosslink with chitosan (usually with protein or amine) to produce blue fluorescent hydrogels, which play a great role in biomedicine and pharmaceutical auxiliaries.

In addition, the most common forms of alginate are its gel and polyvalent metal ions, but in practical application, gels without any polyvalent metal ions are welcome [33]. Recently, Kasak et al. [34] have prepared polyvalent nonmetal alginate fluorescent hydrogels by dynamic complexing method. Crosslinking occurs through the dynamic complexation of 5-oxo-2, 3-dihydro-5H-[1, 3]-thiazolo[3, 2-a] pyridine-3, 7-dicarboxylic acid (TPDCA), carboxylic acid groups in alginate cages and sodium ions (Figure 5). First, different substances, TPDCA and CDs, were synthesized under different external conditions and then crosslinked. The gelation ability and stability of the gels were verified by the gel and inverted tests of stainless steel balls for 16 hours. Among them, molecular fluorophore TPDCA is the key component to initiate gelation, and the existence of fluorophore provides them with bright luminescence.

\section{Types and Properties of Luminescence}

At present, hydrogels can be divided into two types: selfluminescence and non-self-luminescence. Self-luminescence can emit strong fluorescence without other substances.

3.1. Self-Luminescent Hydrogel. Although different covalent bonding and physical embedding methods have been developed to immobilize the fluorophores, their application has been greatly limited due to the complexity of the synthesis route, the potential leakage and photobleaching of the fluorophores, the impact of fluorescent markers on the degradation mode and their application. Unlike non-self-photoluminescence hydrogels, self-luminous hydrogels do not require additional phosphors or fluorophores to label them. In recent years, researchers have immersed many painstaking efforts in the field of unconventional luminescent gel. According to the investigation, a few polymers only contain auxiliary chromophores or unconventional chromophores, such as carbonyl, ester, and amide, which can emit strong fluorescence. At the same time, a small number of clusters can also achieve fluorescence emission and avoid the traditional luminescence defects, that is, aggregation induced quenching (ACQ) effect. On the contrary, it will cause aggregation induced emission (AIE) effect, when the aggregation degree is larger, and the fluorescence is significantly enhanced.

3.1.1. Cluster Luminescence. Cluster luminescence is most common in metal nanoclusters, such as gold nanoclusters, silver nanoclusters, etc. In the gel field, it is not common to include metal nanoclusters, and most of them are based on polymer and small molecule clusters. Based on the completely non conjugated structure of saturated C-C, C-O, or $\mathrm{C}-\mathrm{N}$ bonds, the clusters show bright fluorescence, which is called cluster luminescence [35-38]. In general, with the increase of cluster size, the fluorescence increases. For example, Sugár et al. [39] simulated dimyristoylphosphatidylcholine/distearoylphosphatidylcholine (DMPC/DSPC) lipid bilayer by using Ising model and 


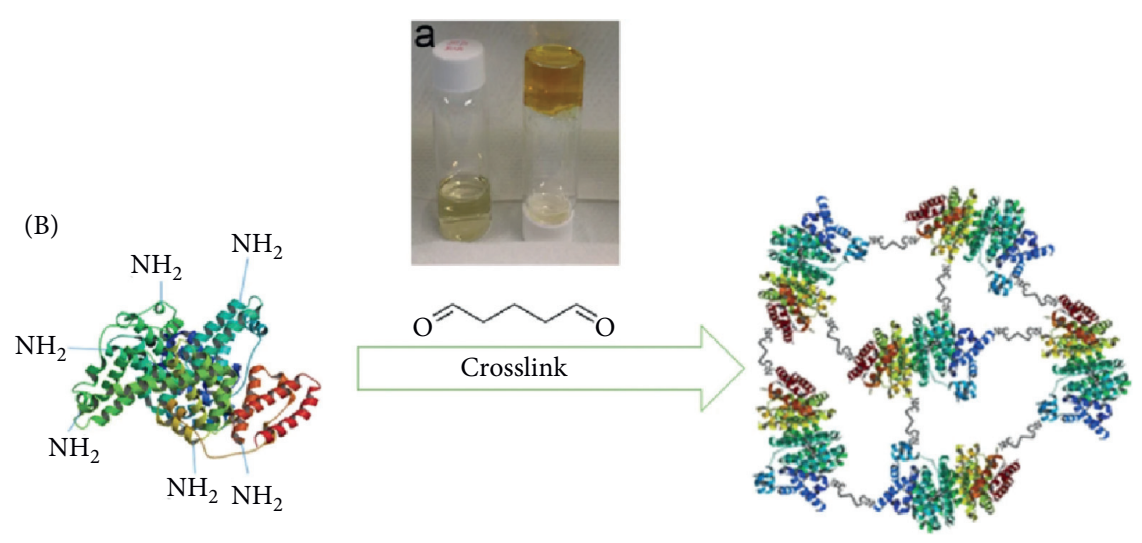

(a)
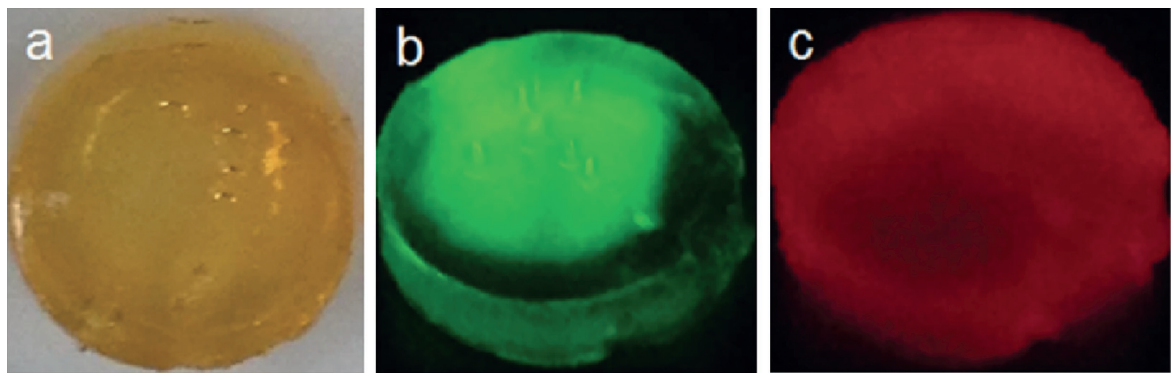

(b)

FIGURE 4: Schematic diagram and macroscopic photoperiod of crosslinked BSA hydrogels. (a) Schematic diagram of crosslinked BSA hydrogels. Among them, the right sample bottle contains BSA hydrogel after crosslinking with GA, while the left sample bottle contains $20 \%$ BSA solution as a comparison. (b) Macroscopic picture of GA crosslinked BSA hydrogel under normal white light, $470 \mathrm{~nm}$ and $595 \mathrm{~nm}$ (reprinted from [29]).

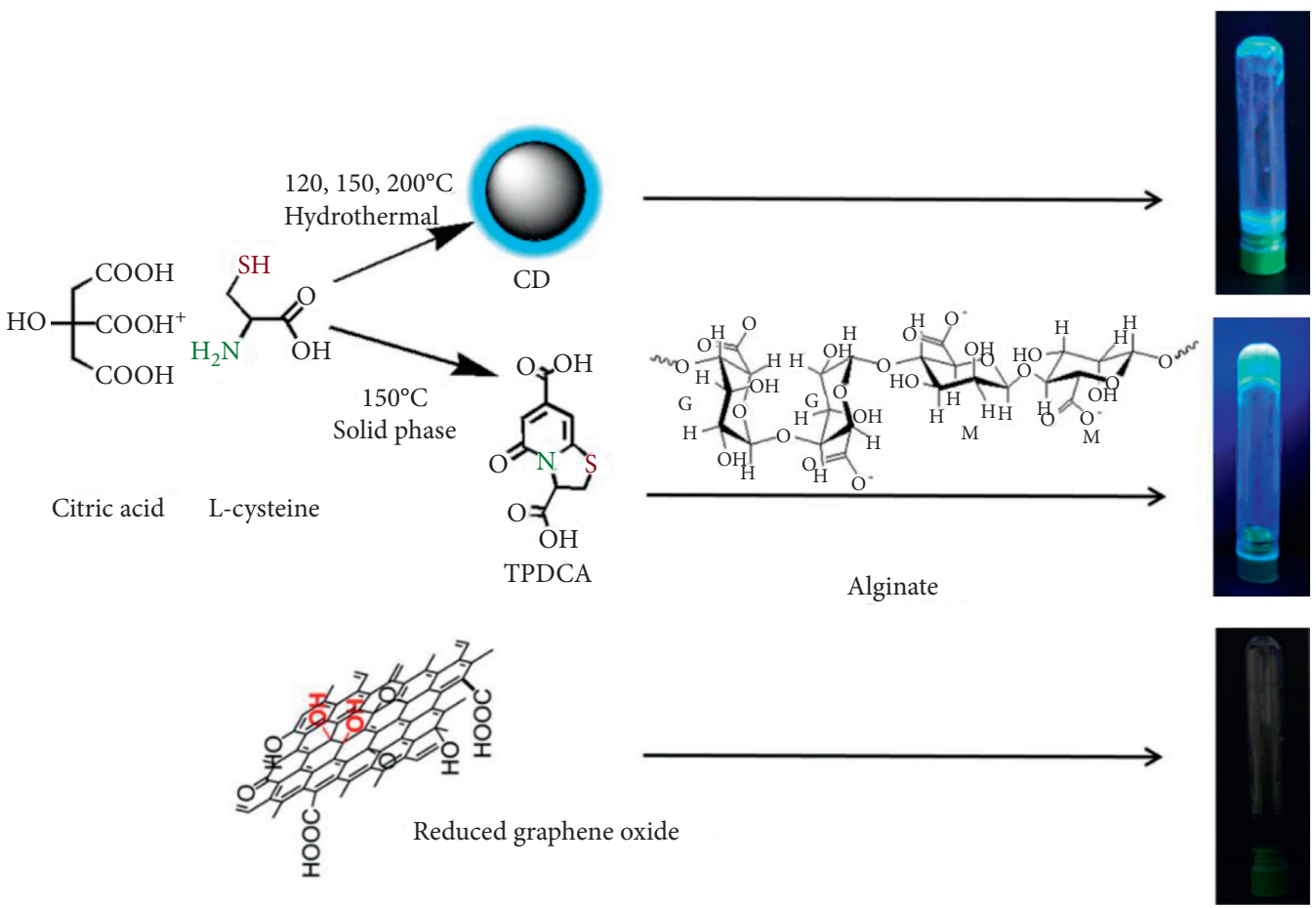

FIGURE 5: Schematic diagram of synthesis of crosslinked alginate fluorescent hydrogels. Among them, the picture on the right was shot under the excitation of ultraviolet lamp (reprinted from [34]). 
Monte Carlo method, and it can be found that there was a strong positive correlation between the permeation threshold temperature of gel cluster and FRAP threshold temperature.

\subsubsection{Unconventional Chromophore Luminescence.} Conventional fluorescent polymers are composed of conjugated main chains or $\pi$ electron systems, which form enough conjugation by covalent bonding, thus producing bright fluorescence. However, their defects, such as poor photostability and high cytotoxicity, limit their application [40]. Due to these limitations, researchers have discovered and designed a series of unconventional luminescent hydrogels. Xu et al. [41] reported the autofluorescence of carbonyl hydrogels. Even without any specific functional groups, polyacrylamide (PAM) gels are autofluorescent. In this work, polymers with carbonyl groups were synthesized from different monomers, and very similar fluorescence could be observed in the gel. Meanwhile, the fluorescence lifetime of the dried PAM gel was measured. The average life expectancy was $1.28 \mathrm{~ns}$, similar to that of the traditional fluorophore containing large conjugated structure. In this work, the factors affecting the fluorescence intensity were studied, and it was found that the fluorescence properties were related to the gel structure. In addition, the water content in the gel also affects the luminous intensity. With the decrease of water content, fluorescence increases gradually. Like traditional fluorescent gel, adding some specific metal ions can quench fluorescence.

3.2. Non-Self-Luminous Hydrogel. Non-self-luminous hydrogel does not have fluorescence emission itself and needs other light emitting materials to achieve fluorescence emission. In general, the organic fluorescent substances, quantum dots, and metal complexes are usually labeled by physical or chemical methods, and then the non-self-luminous hydrogels emit fluorescent quantities.

\subsubsection{Multiresponse Supramolecular Luminescent Hydrogel.} Compared with single response fluorescent gel, multi-response supramolecular luminescent hydrogel can respond to many factors and improve the utilization and sensitivity of fluorescent gel. Therefore, the multi-stimulus responsive fluorescent gel has become the focus of current research [42]. The synthesis of supramolecular luminescent hydrogels by rotaxane with typical mechanical interlocking structure has become a research hotspot. At present, rotaxane usually prepares supramolecular gels with multiple stimulus responses by self-assembly in solution [43]. Sun et al. [44] prepared the first rotaxane with fluorescent functional group based on BODIPY and aromatics. Then, using the rotational alkane as gelation factor, by means of the $\pi-\pi$ interaction between the phenylene parts of the adjacent column aromatic ring of the rotaxane, noncovalent action such as $\pi-\pi$ interaction between BODIPY and imidazolium parts, the 3D crosslinking network is finally self-assembled (Figure 6). Among them, BODIPY is its fluorescent chromophore. By heating-cooling, vibrating-standing at temperature, and adding different anions, the gel can realize reversible gelsolution transformation. At the same time, it also has stimulation response to different $\mathrm{pH}$ values. After being exposed to hydrochloric acid $(\mathrm{HCl})$ gas, the fluorescence intensity of the thin film gel is significantly reduced by $50 \%$, and at the same time, under ultraviolet light, the fluorescence color changes from yellow to purple-red.

Ma research group of Tianjin University [45] reported a fluorescent hydrogel composed of lanthanide and nucleoside. Here, nucleosides, the main component of nucleic acids, act as ligands and successfully chelate with lanthanide elements to form complexes in water. Then, it can self-assemble into fluorescent gel with characteristic luminescence of lanthanide series elements, and the prepared fluorescent hydrogel showed fluorescent photochromic characteristics in response to external stimuli such as $\mathrm{pH}$ value and temperature. Intelligent stimuli responsive hydrogels may undergo reversible deformations when triggering various external stimuli. Cheng and colleagues [46] proposed a double-layer hydrogel actuator with fluorescent coumarin groups. Under the stimulation of $\mathrm{pH}$ or temperature, the double-layer hydrogel shows reversible deformation behavior, and the whole hydrogel has bright fluorescence due to the introduction of coumarin units. In conclusion, the research progress of multi-response supramolecular luminescent hydrogels has always been a hot topic.

3.2.2. White Luminescent Hydrogel. White light emitting (WLE) hydrogel has attracted considerable attention in the manufacture of intelligent molecular machines, equipment, luminescent lamps, and materials [47].

Xia et al. [11] reported a fluorescent hydrogel based on peptide motif and transition metal ion assembly. The introduction of metal ions to immobilize them on the hydrogel network at specific locations can prevent the aggregation and self-quenching of chromophores. In addition, white light emission is realized by using three different metal ions (Figure 7). Because the emission spectra of $\mathrm{Eu}^{3+}, \mathrm{Ru}^{2+}$, and $\mathrm{Ir}^{2+}$ are complementary in the visible range, $\mathrm{Eu}^{3+}, \mathrm{Ru}^{2+}$, and $\mathrm{Ir}^{2+}$ are selected here to form fluorescent metal complexes with EFK-Bpy. The emission spectra of EFK-Bpy-Eu, EFKBpy-Ir, and EFK-Bpy-Ru hydrogel excited by $365 \mathrm{~nm}$ can cover the whole visible range. Therefore, white light emission can be easily realized by adjusting the proportion of the three components.

Lanthanide has always been popular in the field of luminescence because of its good luminescent properties, even though it is expensive. Recently, Xue et al. [48] reported a pure white emissive hydrogel composed of luciferin and carbon dots with high quantum yield. By adjusting the stoichiometry of lanthanide complexes, fluorescein and carbon dots, a pure white emitting hydrogel can be obtained. The absolute yield of WLE hydrogel was up to $18.00 \%$ by computation.

\subsubsection{Reversible Fluorescent Chromotropic Hydrogel.} More and more attention has been paid to the development of advanced sensors and luminescent materials, which are 


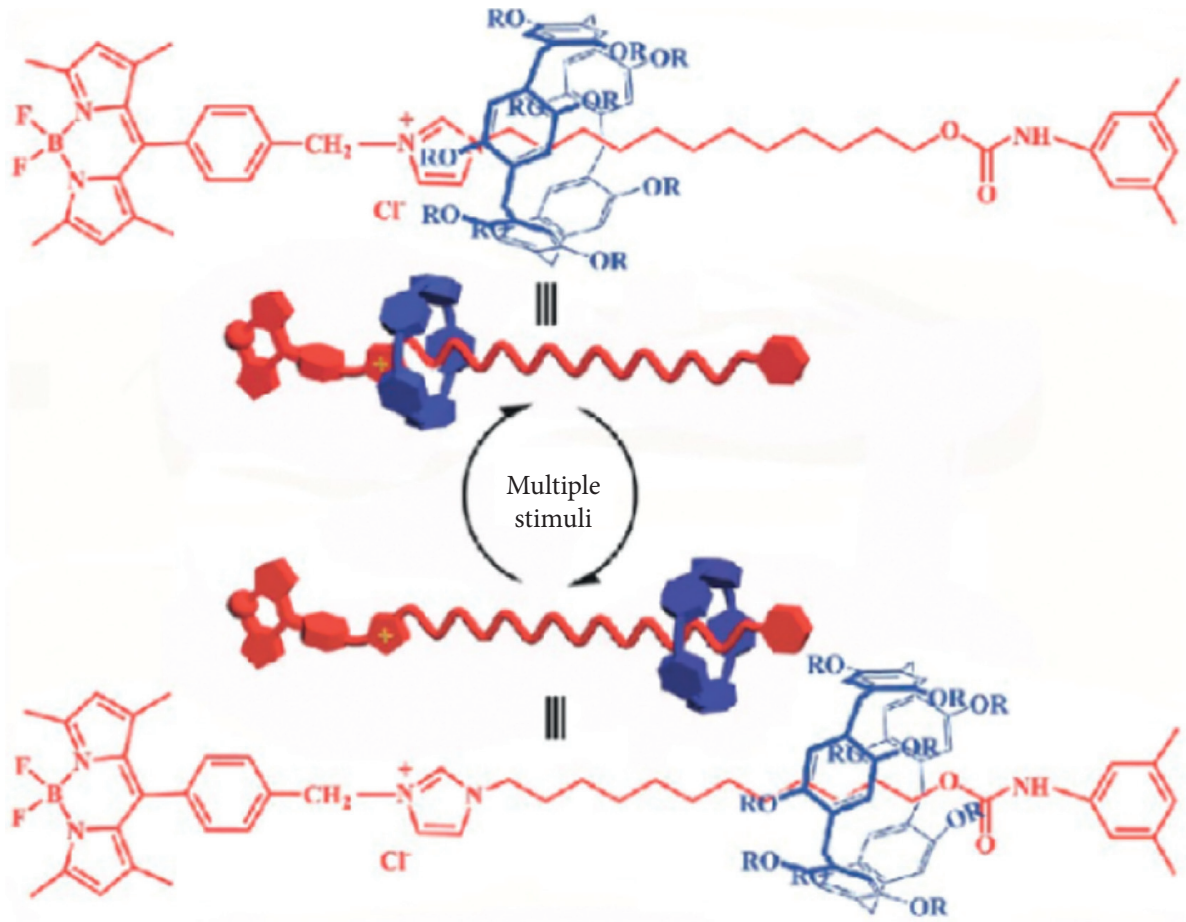

(a)

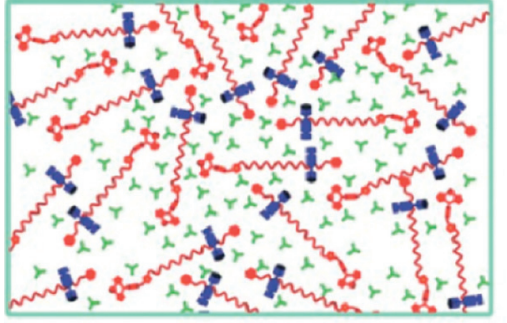

Solution

מamm- [2] rotaxane BC12P5

y DMSO

nnm Fibers
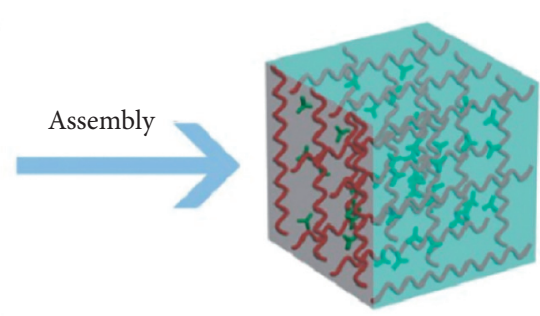

Gel

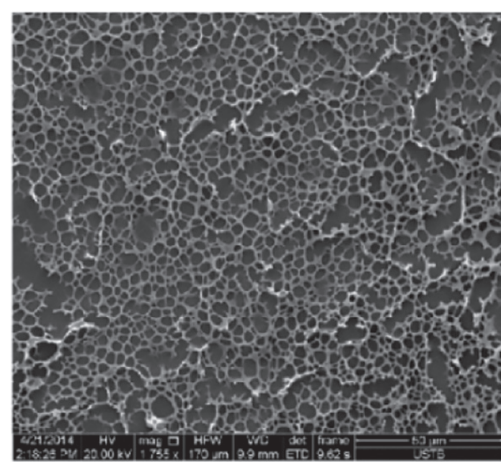

(c)

Figure 6: Schematic diagram and gel SEM diagram of the wheel molecule and gel structure. (a) The structure of rotaxane and the schematic diagram of its structural transformation under various stimuli. (b) Schematic diagram of self-assembly of BC12P5 in supramolecular gels. (c) SEM images of rotan-based supramolecular gels formed in DMSO (reprinted from [44]).

dynamically changing in response to external stimuli $[49,50]$. Reversible fluorescent chromotropic hydrogel is also used as a kind of fluorescent gel. Yang et al. reported a work based on polymer hydrogel containing lanthanide elements and prepared reversible fluorescent color-changing gel by using lanthanide-mannose compound in gel matrix [51]. Among them, mannose, as a ligand, coordinated with lanthanide ions $\left(\mathrm{Eu}^{3+}\right.$ and $\left.\mathrm{Tb}^{3+}\right)$ in aqueous solution, sensitized rare Earth ions to emit light, and first formed lanthanide mannose (Ln-Man) complex. Subsequently, gelatin network was introduced into the system to prepare the rare Earth hydrogel successfully (Figure 8 ). The prepared fluorescent gel has fluorescence discoloration function for $\mathrm{Fe}^{2+}$, and the recovery rate of fluorescent discoloration is $63.2 \%$ after three cycles of "ON/OFF." Despite the decrease of fluorescence intensity, the "ON/OFF" switchable emission is reversible in response to $\mathrm{Fe}^{2+}$ and edetic acid (EDTA) due to the dynamic coordination between lanthanide and mannose. Because of its good biocompatibility, the gel can be used as a $3 \mathrm{D}$ cell culture medium and still has a reversible "ON/OFF" fluorescence characteristic in the matrix.

\subsubsection{Color Matching Intrinsic Luminescence Metal-Organic} Frameworks (MOF) Gel. In recent years, metal-organic framework has been a new material which attracts much attention. As for the tintable intrinsic luminescent MOF gel, it has been developed as early as 2018. Chen group of Nankai University [50] reported for the first time a series of coloring luminescent lanthanide MOF gel materials. Lanthanide metal ions and organic ligands with unevenly 

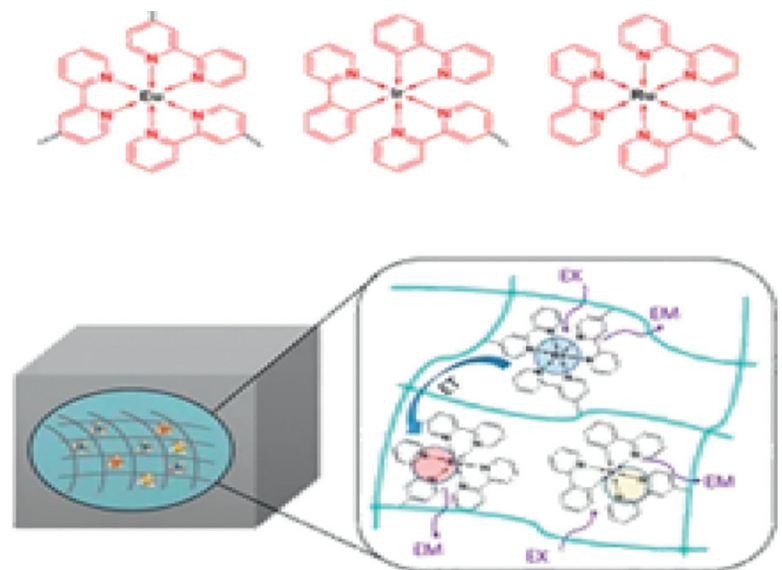

(a)

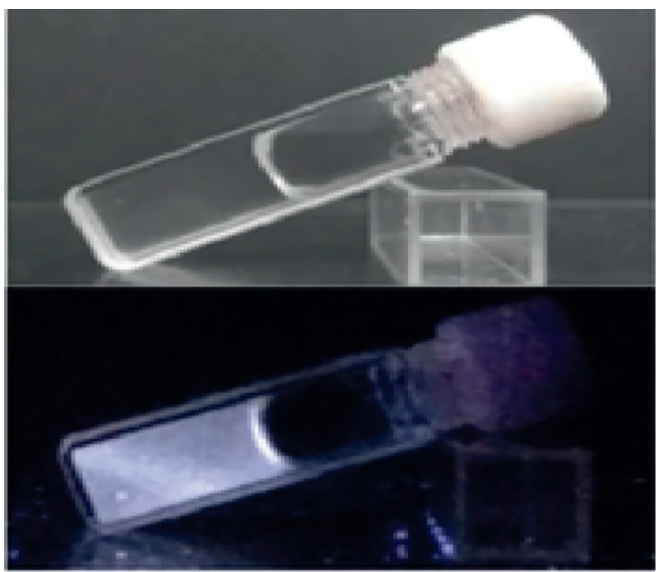

(b)
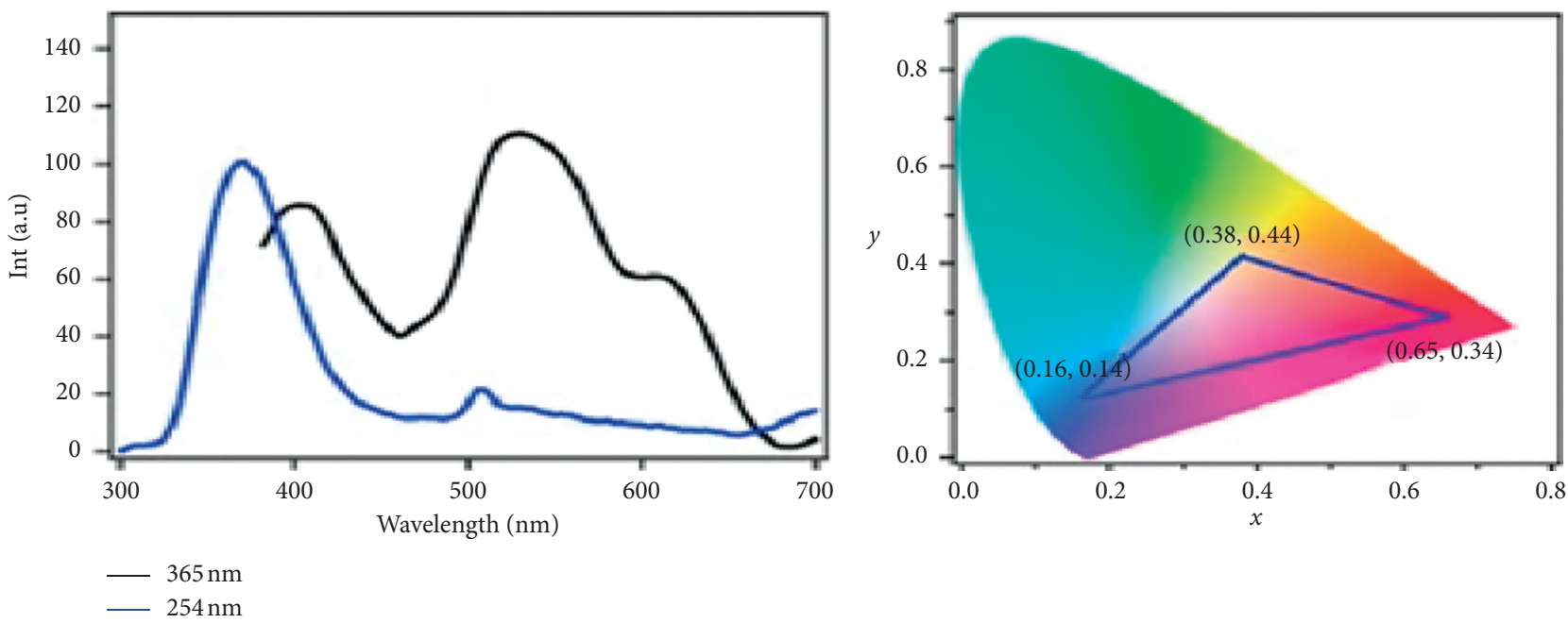

(c)

(d)

Figure 7: White light emission mechanism. (a) The schematic diagram of the hydrogel was co-assembled. (b) Photo of WLFH (12 mm) illuminated by natural light (top) and UV 1 (bottom). (c) Emission spectrum of WLFH ( $\lambda \mathrm{ex}=365 \mathrm{~nm}, \lambda \mathrm{ex}=254 \mathrm{~nm})$. (d) Color chart of EFK-Bpy-Eu, EFK-Bpy-Ir, and EFK-Bpy-Ru hydrogel (reprinted from [11]).
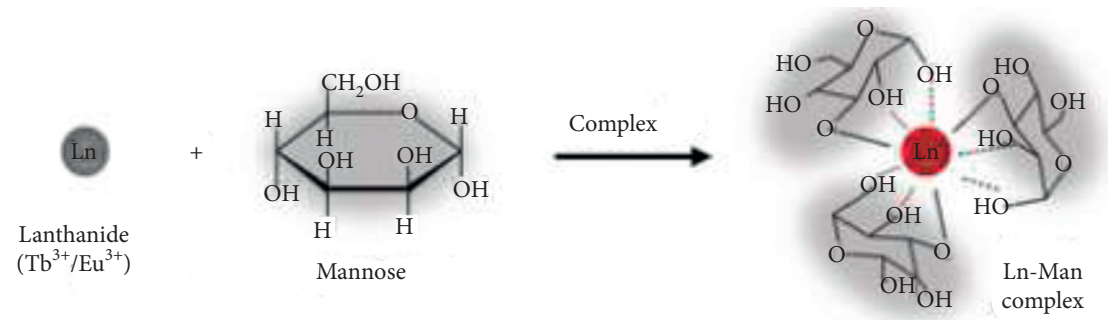

(a)

Figure 8: Continued. 


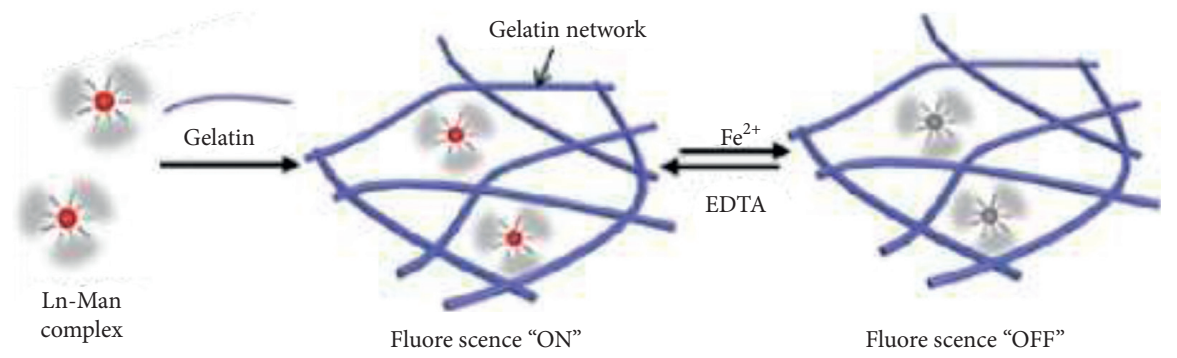

(b)
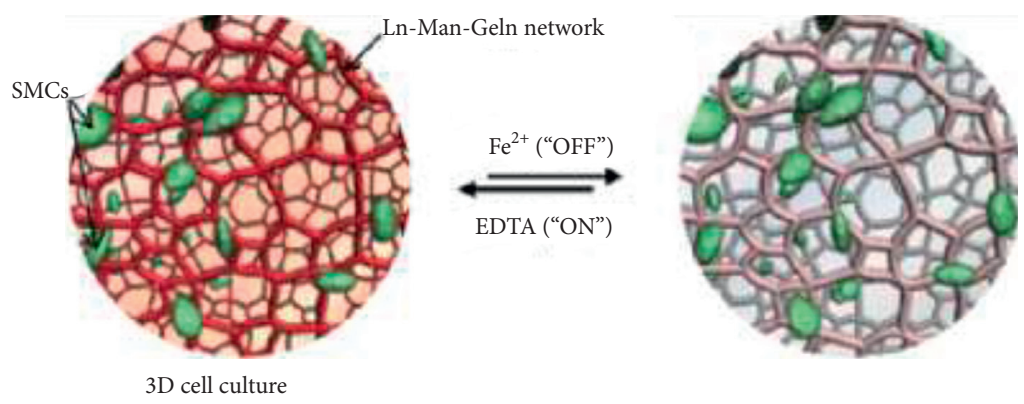

(c)

Figure 8: Gel formation and its application as a substrate for 3D cell culture. (a) The formation of lanthanide mannose (Ln-Man) complex. (b) The formation of lanthanum mannose gelatin (Ln-Man-Geln) hydrogel and the fluorescence "ON/OFF" on Fe ${ }^{2+} / \mathrm{EDTA}$. (c) Ln-ManGeln hydrogel as a schematic diagram of 3D cell culture medium (reprinted from [51]).

distributed carboxyl groups were selected. Nanobelts were formed through the high valence and biased carboxyl distribution of $\mathrm{Ln}^{3+}$ ions, boric acid groups were introduced to improve anisotropic growth through steric hindrance and hydrogen bonding, and then these nanobelts were wound together and gelled to form MOF gel (Figure 9(c)). Among them, the addition of boron in the side chain of Isophthalic acid (ISP) changed the ligand's entropy, hydrogen bond, and steric hindrance, which played an important role in the formation of MOF gel. Monometallic MOF shows red, green, and blue fluorescence. Therefore, mixed metal MOF gel was prepared by adjusting the type and/or proportion of $\mathrm{Ln}^{3+}$ ions; single wavelength excitation can make it obtain full-color luminescence (Figure 9(a)). As an excellent chemical/ physical hybrid gel with excellent optical properties, it will have great potential in the field of target detection and biosensing.

\section{Study on the Application of Fluorescent Hydrogel}

Luminescent materials have attracted considerable attention due to their wide potential applications, especially hydrogels. Based on the noncovalent interaction of gel factor, soft materials, which have highly swelling, degradable, and adjustable rheological properties, are more convenient for synthesis and preparation methods, responsive to external stimuli, and have unique physical properties and selfrepairing properties. The special properties of these unique structures make them have great potential in sensors, fluorescence imaging, molecular devices, and biological therapy.
4.1. Sensors. Fluorescence-based sensors have been routinely used in biology, physiology, pharmacology, and environmental science. Because of their physiological compatibility, fluorescent gel sensors have attracted wide attention in medicine. Diabetes is a very common chronic disease at present, which is accompanied by various complications, and it is particularly necessary to detect and observe it in real time.

Park and Park [52] reported a glucose sensor with fluorescence and size changing dual response, which was fabricated by microfluidics. Poly(acrylamide) (PAAm) hydrogel forms PAAc hydrogel under the treatment of sodium hydroxide and tetramethylethylenediamine (TEMED). Finally, gelatin was modified to combine fluorescent CDs, glucose oxidase (GOx), horseradish peroxidase (HRP), and gel droplets. The reaction of GOx and HRP with glucose will produce gluconic acid and $-\mathrm{OH}$ radicals, which will lead to fluorescence quenching and size reduction of CDs, respectively. Moreover, the glucose sensor shows good sensitivity (linear range is about $30 \mathrm{~mm}$; detection limit is $0.052 \mathrm{~mm}$ ). Because of its good biocompatibility, it has no other side effects on organisms, so it is of great significance to use it as a long-term glucose test.

Lanthanide-based sensors have unique spectral properties such as long fluorescence lifetime and large Stokes shift. Therefore, many people love to use it to make fluorescent gel for sensor [53]. Weng et al. [54] reported fluorescence hydrogels based on Eu-Iminodiacetate (Eu-IDA dynamic) coordination, which can be dynamically broken and bonded under $\mathrm{pH}$, temperature, metal ions, ultrasonic treatment, and various force stimuli and can switch light device and sol-gel 


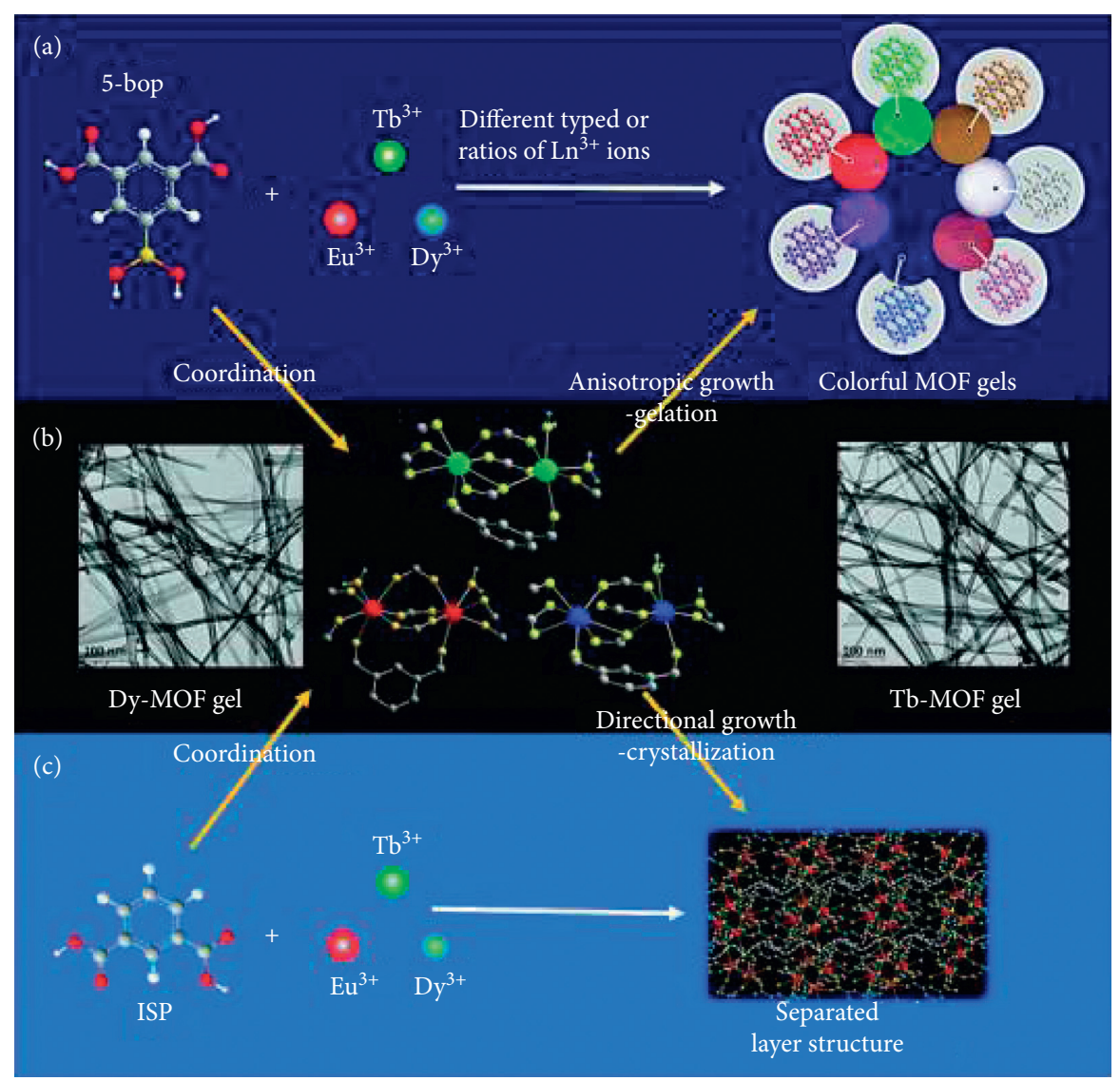

FIGURE 9: Formation and photoluminescence of chemical/physical hybrid gel with intrinsic fluorescence emission. (a) Choosing different types and proportions of $\mathrm{Ln}^{3+}$ ions to form MOF gel with different colors. (b) Formation of Dy-MOF and Tb-MOF. (c) Formation of MOF gel (reprinted from [50]).

trasition easily. Intelligent optical materials can respond to a variety of stimuli, which provides a new method for the development of multi-responsive biosensors.

4.2. Biological Imaging Probe. Prior to this, Mehwish et al. [55] summarized the supramolecular fluorescent hydrogels used as biological imaging probes in detail. This includes the research of preparation methods, excellent fluorescence properties, prospective applications in biological imaging, and opportunities and challenges in the future and makes a detailed classification and description of the imaging mechanism of different types of fluorescent probes. The team points out that the supramolecular gel fluorescent probes are highly specific and have excellent stability and sensitivity. At the same time, molecules are assembled by noncovalent bonds, such as hydrogen bonds and hydrophobic interactions, which makes the imaging method more physiological and have good biocompatibility. Therefore, fluorescent gel has a larger market in the application field of biological imaging probes.

Subsequently, Xu et al. [56] reported a fluorescent hydrogel probe for monosaccharide detection. The anthracene fluorescence probe based on boric acid was functionalized with acrylamide unit to incorporate into the hydrogel system for monosaccharide detection (Figure 10). The fluorescence probe showed a strong fluorescence opening response after being exposed to fructose. According to the fluorescence response, the sensitivity of the probe was improved 10 times by adding D-fructose, and it showed excellent monosaccharide detection ability. Recently, the application of hydrogel fluorescent probes has not been reduced and is still in active research and development [57-59].

4.3. Drug Delivery. Using appropriate drug delivery system can ensure higher bioavailability of drugs, and it can exert its efficacy for a long time. Fluorescent gel with good biocompatibility and biodegradability and no toxic and side effects has become a suitable material [60, 61]. The sulfhydryl-disulfide bond is easily broken in the cell, resulting in the destruction of the nanomaterials 3D network, and further accurately releasing the drug. Therefore, in the redox drug delivery system, the disulfide bond has been the most commonly used functional group to prepare nanogels to achieve controlled release of drugs in cancer cells [62]. At the same time, the design of nanogels with redox response and aggregation induced emission luminescence (AIEgen) function can achieve accurate and traceable delivery of drugs.

Ma et al. [63] reported a multifunctional nanomel gel project with redox reaction and AIEgen characteristics. 


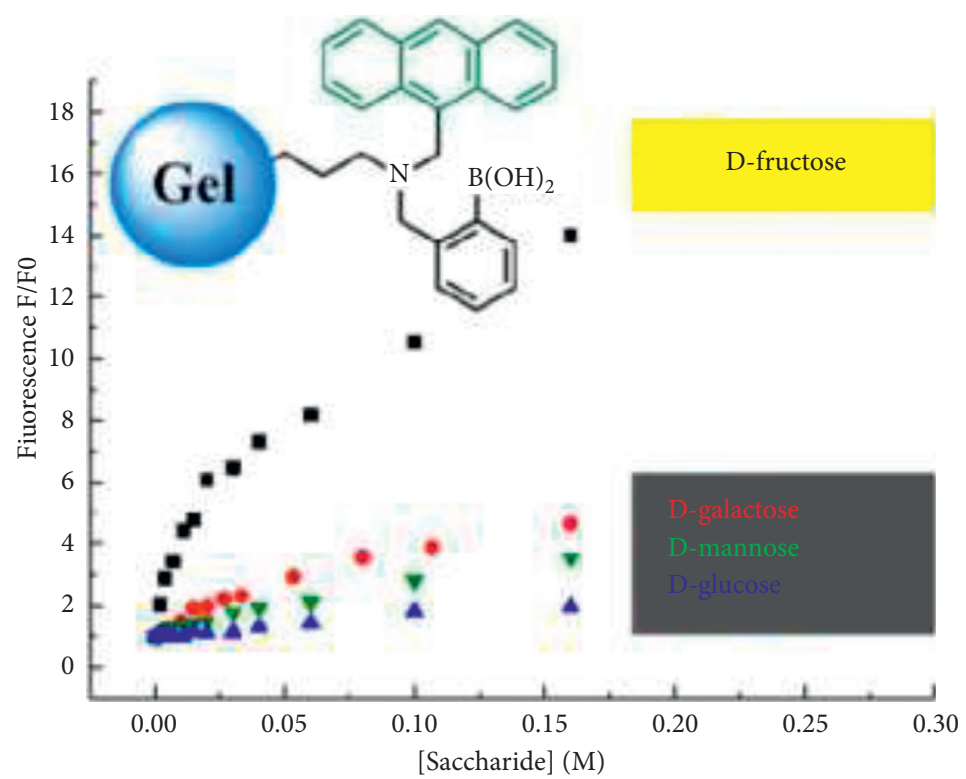

(a)
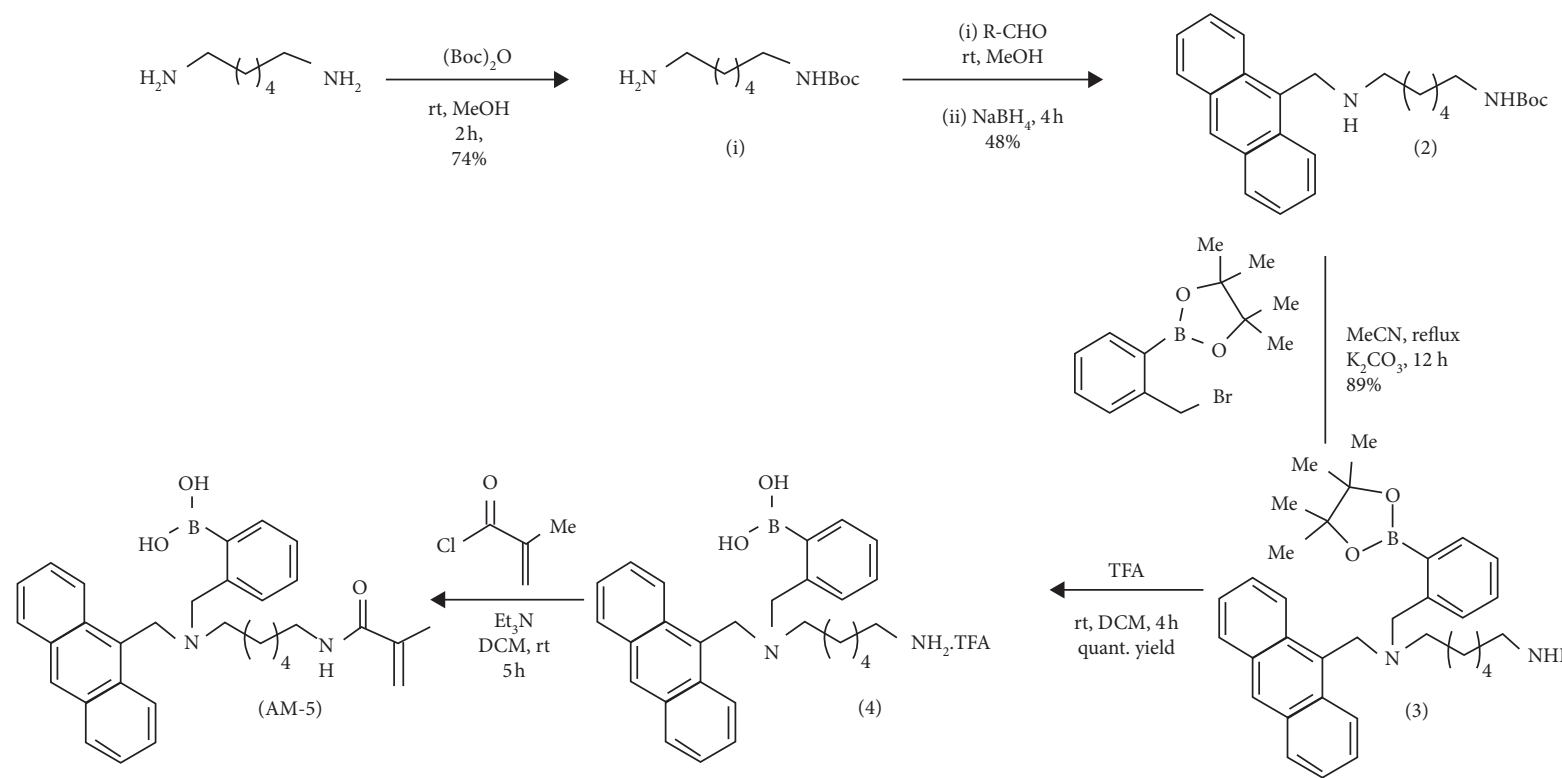

(b)

FIGURE 10: The detection of monosaccharide and the route of gel probe synthesis. (a) Schematic diagram of monosaccharide detection based on fluorescence. (b) Synthesis of boric acid fluorescent probes (reprinted from [56]).

All-transretinoic acid (ATRA), with a disulfide bond as a linking agent and a group of aggregation induced luminescence $\left(\mathrm{TPENH}_{2}\right)$ grafted with hyaluronic acid $(\mathrm{HA})$, forms a fluorescent gel HA-ss-ATRA/TPENH ${ }_{2} \mathrm{HNPs}$ delivery carrier: ATRA as the hydrophobic core of multifunctional hyaluronic acid nanomaterials (HNPs), HA as a hydrophilic agent, using disulfide bond as redox sensitive bonds between HA and ATRA, and preparing HA-ss-ATRA two affinity conjugates, which can control the release of drugs according to the intracellular environment (Figure 11). Finally, HA-ss-ATRA, dichloroethane (EDC), and N-hydroxysuccinimide (NHS) were co-dissolved in formamide to modify AIE fluorescence group. With the successful preparation of HNPs with unique AIE characteristics, adriamycin loaded on them is selectively absorbed by cancer cells, and real-time imaging in dynamic environment is realized.

4.4. Other Applications. Fluorescent gel has excellent water content and unique luminescent properties. It can be used as a sensor and effectively adsorb heavy metals and other environmental pollutants $[64,65]$.

At present, fluorescence hydrogels are used to encrypt and protect data information so as to realize the possibility of multi-stage data security. Zhang research group of 


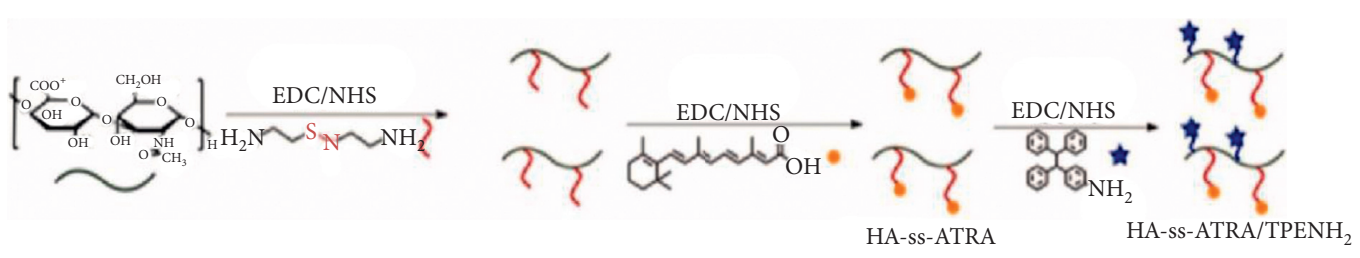

(a)
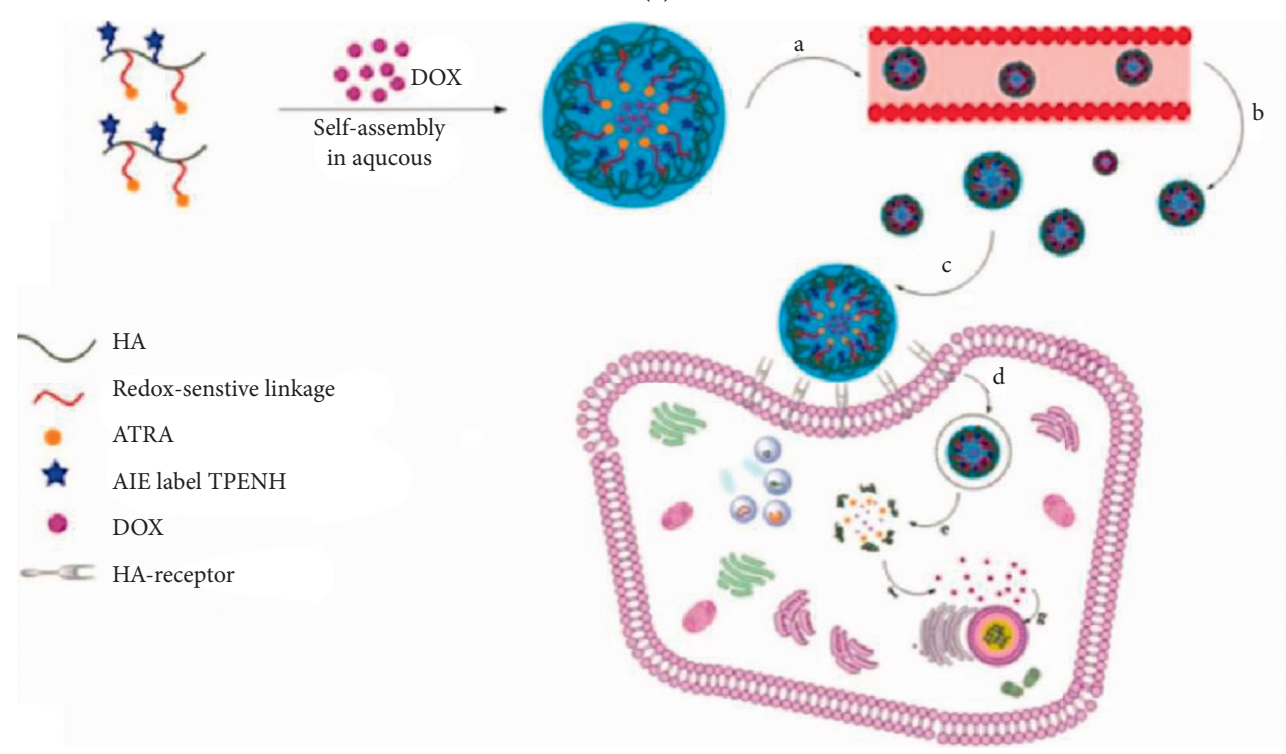

(b)

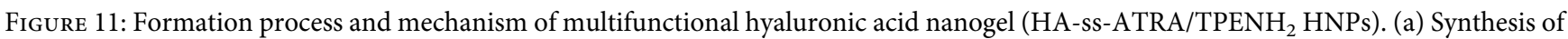
HA-ss-ATRA/TPENH ${ }_{2}$ conjugate. (b) HA-ss-ATRA/TPENH ${ }_{2}$ HNPs is formed and used for drug delivery (reprinted from [63]).

Ningbo Institute of Materials [66] showed a 3D based anticounterfeit platform based on fluorescent hydrogel, shape memory, and self-repair feature and made full use of the data inside the $3 \mathrm{D}$ structure to encrypt. At the same time, the research group [67] reported a safe information storage method, which can store information on fluorescent hydrogel based on controllable ion imprinting. When the 2D information of fluorescence quenching and the 3D information of activation are exposed to ultraviolet rays and put into water, it can be decrypted in layers and dimensions. Fluorescent gel can also be used to manufacture special materials [68]. Due to its excellent self-healing function, fluorescent hydrogels can also be used as medical excipients in biomedicine $[69,70]$. In a word, the function of fluorescent hydrogel is reflected in many aspects, and there will be more room for further application in the future [71-77].

\section{Conclusion}

The hydrophilic functional groups attached to the polymer backbone make the gel have high water absorbency and unique 3D network. The fluorescent hydrogels with good biocompatibility and luminescence properties show good application prospects in biomedicine. At present, the fluorescent hydrogels have been widely used in sensors, biological imaging probes, and so on. In this review, three different preparation methods are introduced, which are used to construct fluorescent hydrogels with excellent properties. However, the fluorescent hydrogels also have some defects. For example, the gel prepared by noncovalent interaction has poor mechanical stability due to the weak interaction between the gels. Therefore, based on the good prospect of fluorescent hydrogel, accelerating the perfection of fluorescent gel defects and preparing better fluorescent hydrogels are particularly important.

\section{Data Availability}

The data used to support the findings of this study are included within the article.

\section{Conflicts of Interest}

The authors declare no conflicts of interest.

\section{Acknowledgments}

The authors greatly appreciate the financial support of the National Natural Science Foundation of China (Nos. 21872119 and 22072127), the Talent Engineering Training Funding Project of Hebei Province (No. A201905004), and the Research Program of the College Science and Technology of Hebei Province (No. ZD2018091). 


\section{References}

[1] E. M. Ahmed, "Hydrogel: preparation, characterization, and applications: a review," Journal of Advanced Research, vol. 6, no. 2, pp. 105-121, 2015.

[2] E. Caló and V. V. Khutoryanskiy, "Biomedical applications of hydrogels: a review of patents and commercial products," European Polymer Journal, vol. 65, pp. 252-267, 2015.

[3] A. P. Demchenko, "The red-edge effects: 30 years of exploration," Luminescence, vol. 17, no. 1, pp. 19-42, 2002.

[4] A. Griffith, T. J. Bandy, M. Light, and E. Stulz, "Fluorescent hydrogel formation from carboxyphenyl-terpyridine," Chemical Communications, vol. 49, no. 7, pp. 731-733, 2013.

[5] A. Khabibullin, M. Alizadehgiashi, N. Khuu, E. Prince, M. Tebbe, and E. Kumacheva, "Injectable shear-thinning fluorescent hydrogel formed by cellulose nanocrystals and graphene quantum dots," Langmuir, vol. 33, no. 43, pp. 12344-12350, 2017.

[6] T. H. Kim, J. Seo, S. J. Lee, S. S. Lee, J. Kim, and J. H. Jung, "Strongly fluorescent hydrogel as a blue-emitting nanomaterial: an approach toward understanding fluorescence-structure relationship," Chemistry of Materials, vol. 19, no. 24, pp. 5815-5817, 2007.

[7] J. T. Suri, D. B. Cordes, F. E. Cappuccio, R. A. Wessling, and B. Singaram, "Continuous glucose sensing with a fluorescent thin-film hydrogel," Angewandte Chemie-International Edition, vol. 42, no. 17, pp. 5857-5859, 2003.

[8] B. Tan, H. Zhao, L. Du, X. Gan, and X. Quan, "A versatile fluorescent biosensor based on target-responsive graphene oxide hydrogel for antibiotic detection," Biosensors and Bioelectronics, vol. 83, pp. 267-273, 2016.

[9] K. Ariga, J. P. Hill, M. V. Lee, A. Vinu, R. Charvet, and S. Acharya, "Challenges and breakthroughs in recent research on self-assembly," Science and Technology of Advanced Materials, vol. 9, no. 1, Article ID 014109, 2008.

[10] G. M. Whitesides and B. Grzybowski, "Self-assembly at all scales," Science, vol. 295, no. 5564, pp. 2418-2421, 2002.

[11] Y. Xia, B. Xue, M. Qin, Y. Cao, Y. Li, and W. Wang, "Printable fluorescent hydrogels based on self-assembling peptides," Scientific Reports, vol. 7, Article ID 9691, 2017.

[12] G. von Maltzahn, S. Vauthey, S. Santoso, and S. Zhang, "Positively charged surfactant-like peptides self-assemble into nanostructures," Langmuir, vol. 19, no. 10, pp. 4332-4337, 2003.

[13] X. Zhao and S. Zhang, "Molecular designer self-assembling peptides," Chemical Society Reviews, vol. 35, no. 11, pp. 1105-1110, 2006.

[14] R. V. Ulijn and A. M. Smith, "Designing peptide based nanomaterials," Chemical Society Reviews, vol. 37, no. 4, pp. 664-675, 2008.

[15] N. T. Chu, R. D. Chakravarthy, N. C. Shih et al., "Fluorescent supramolecular hydrogels self-assembled from tetraphenylethene (TPE)/single amino acid conjugates," RSC Advances, vol. 8, no. 37, pp. 20922-20927, 2018.

[16] J. J. Yan, H. Wang, Q. H. Zhou, and Y. Z. You, "Reversible and multisensitive quantum dot gels," Macromolecules, vol. 44, no. 11, pp. 4306-4312, 2011

[17] T. Y. Cheng, M. H. Chen, W. H. Chang, M. Y. Huang, and T. W. Wang, "Neural stem cells encapsulated in a functionalized self-assembling peptide hydrogel for brain tissue engineering," Biomaterials, vol. 34, no. 8, pp. 2005-2016, 2013.

[18] X. F. Ji, P. Wang, H. Wang, and F. H. Huang, "A fluorescent supramolecular crosslinked polymer gel formed by crown ether based host-guest interactions and aggregation induced emission," Chinese Journal of Polymer Science, vol. 33, no. 6, pp. 890-898, 2015.

[19] C. W. Zhang, B. Ou, S. T. Jiang et al., "Cross-linked AIE supramolecular polymer gels with multiple stimuli-responsive behaviours constructed by hierarchical self-assembly," Polymer Chemistry, vol. 9, no. 15, pp. 2021-2030, 2018.

[20] S. Bhowmik, S. Banerjee, and U. Maitra, "A self-assembled, luminescent europium cholate hydrogel: a novel approach towards lanthanide sensitization," Chemical Communications, vol. 46, no. 45, pp. 8642-8644, 2010.

[21] T. Katashima, U. i. Chung, and T. Sakai, "Mechanical properties of doubly crosslinked gels," Polymer Journal, vol. 51, no. 9, pp. 851-859, 2019.

[22] D. C. Schoenmakers, A. E. Rowan, and P. H. J. Kouwer, "Crosslinking of fibrous hydrogels," Nature Communications, vol. 9, Article ID 2172, 2018.

[23] H. Shibata, Y. J. Heo, T. Okitsu, Y. Matsunaga, T. Kawanishi, and S. Takeuchi, "Injectable hydrogel microbeads for fluorescence-based in vivo continuous glucose monitoring," Proceedings of the National Academy of Sciences, vol. 107, no. 42, pp. 17894-17898, 2010.

[24] T. Nishiyama, Y. Kagami, T. Yamauchi, and N. Tsubokawa, "Preparation of stimulus-sensitive gel particles with a DNAdye complex and their $\mathrm{pH}$ sensitivity," Polymer Journal, vol. 44, no. 5, pp. 396-400, 2012.

[25] M. Chen, S. Deng, Y. Gu, J. Lin, M. J. MacLeod, and J. A. Johnson, "Logic-controlled radical polymerization with heat and light: multiple-stimuli switching of polymer chain growth via a recyclable, thermally responsive gel photoredox catalyst," Journal of the American Chemical Society, vol. 139, no. 6, pp. 2257-2266, 2017.

[26] H. Wang, J. Di, Y. Sun et al., "Biocompatible PEG-chitosan@ carbon dots hybrid nanogels for two-photon fluorescence imaging, near-infrared light/pH dual-responsive drug carrier, and synergistic therapy," Advanced Functional Materials, vol. 25, no. 34, pp. 5537-5547, 2015.

[27] C. H. Zhu, Z. B. Hai, C. H. Cui, H. H. Li, J. F. Chen, and $\mathrm{S}$. $\mathrm{H}$. $\mathrm{Yu}$, "In situ controlled synthesis of thermosensitive poly(N-isopropylacrylamide)/Au nanocomposite hydrogels by gamma radiation for catalytic application," Small, vol. 8, no. 6, pp. 930-936, 2012.

[28] M. Kawa and T. Takahagi, "Improved antenna effect of terbium(III)-cored dendrimer complex and green-luminescent hydrogel by radical copolymerization," Chemistry of Materials, vol. 16, no. 11, pp. 2282-2286, 2004.

[29] X. Ma, X. Sun, D. Hargrove et al., "A biocompatible and biodegradable protein hydrogel with green and red autofluorescence: preparation, characterization and in vivo biodegradation tracking and modeling," Scientific Reports, vol. 6, Article ID 19370, 2016.

[30] M. E. Nimni, D. Cheung, B. Strates, M. Kodama, and K. Sheikh, "Chemically modified collagen: a natural biomaterial for tissue replacement," Journal of Biomedical Materials Research, vol. 21, no. 6, pp. 741-771, 1987.

[31] A. J. Habeeb and R. Hiramoto, "Reaction of proteins with glutaraldehyde," Archives of Biochemistry and Biophysics, vol. 126, no. 1, pp. 16-26, 1968.

[32] R. A. A. Muzzarelli, "Genipin-crosslinked chitosan hydrogels as biomedical and pharmaceutical aids," Carbohydrate Polymers, vol. 77, no. 1, pp. 1-9, 2009.

[33] M. M. Pérez-Madrigal, J. Torras, J. Casanovas, M. Häring, C. Alemán, and D. D. Díaz, "Paradigm shift for preparing versatile $\mathrm{M}^{2+}$-free gels from unmodified sodium alginate," Biomacromolecules, vol. 18, no. 9, pp. 2967-2979, 2017. 
[34] P. Kasak, M. Danko, S. Zavahir et al., "Identification of molecular fluorophore as a component of carbon dots able to induce gelation in a fluorescent multivalent-metal-ion-free alginate hydrogel," Scientific Reports, vol. 9, Article ID 15080, 2019.

[35] X. Zhou, W. Luo, H. Nie et al., "Oligo(maleic anhydride)s: a platform for unveiling the mechanism of clusteroluminescence of non-aromatic polymers," Journal of $\mathrm{Ma}$ terials Chemistry C, vol. 5, no. 19, pp. 4775-4779, 2017.

[36] E. Zhao, J. W. Y. Lam, L. Meng et al., "Poly[(maleic anhydride)-alt-(vinyl acetate)]: a pure oxygenic nonconjugated macromolecule with strong light emission and solvatochromic effect," Macromolecules, vol. 48, no. 1, pp. 64-71, 2015.

[37] H. Zhang, Z. Zhao, P. R. McGonigal et al., "Clusterizationtriggered emission: uncommon luminescence from common materials," Materials Today, vol. 32, pp. 275-292, 2020.

[38] R. Hu, N. L. C. Leung, and B. Z. Tang, "AIE macromolecules: syntheses, structures and functionalities," Chemical Society Reviews, vol. 43, no. 13, pp. 4494-4562, 2014.

[39] I. P. Sugár, E. Michonova-Alexova, and P. Lee-Gau Chong, "Geometrical properties of gel and fluid clusters in DMPC/ DSPC bilayers: Monte Carlo simulation approach using a two-state model," Biophysical Journal, vol. 81, no. 5, pp. 2425-2441, 2001.

[40] X. Guan, D. Zhang, T. Jia et al., "Unprecedented strong photoluminescences induced from both aggregation and polymerization of novel nonconjugated $\beta$-cyclodextrin dimer," Industrial \& Engineering Chemistry Research, vol. 56, no. 14, pp. 3913-3919, 2017.

[41] H.-X. Xu, Y. Tan, D. Wang et al., "Autofluorescence of hydrogels without a fluorophore," Soft Matter, vol. 15, no. 17, pp. 3588-3594, 2019.

[42] S. Wei, W. Lu, X. Le et al., "Bioinspired synergistic fluorescence-color-switchable polymeric hydrogel actuators," Angewandte Chemie International Edition, vol. 58, no. 45, pp. 16243-16251, 2019.

[43] Y. Liu, H. Wang, P. Liang, and H. Y. Zhang, "Water-soluble supramolecular fullerene assembly mediated by metallobridged $\beta$-cyclodextrins," Angewandte Chemie International Edition, vol. 43, no. 20, pp. 2690-2694, 2004.

[44] N. Sun, X. Xiao, W. Li, and J. Jiang, "Multistimuli sensitive behavior of novel bodipy-involved pillar 5 arene-based fluorescent 2 rotaxane and its supramolecular gel," Advanced Science, vol. 2, no. 9, Article ID 1500082, 2015.

[45] Q. Ma, M. Zhang, X. Xu et al., "Multiresponsive supramolecular luminescent hydrogels based on a nucleoside/lanthanide complex," ACS Applied Materials \& Interfaces, vol. 11, no. 50, pp. 47404-47412, 2019.

[46] Y. Cheng, K. Ren, D. Yang, and J. Wei, "Bilayer-type fluorescence hydrogels with intelligent response serve as temperature/pH driven soft actuators," Sensors and Actuators B: Chemical, vol. 255, pp. 3117-3126, 2018.

[47] Z. Wang, B. Chen, A. S. Susha et al., "All-copper nanocluster based down-conversion white light-emitting devices," $A d$ vanced Science, vol. 3, no. 11, Article ID 1600182, 2016.

[48] J. Xue, X. Xu, Y. Zhu, and D. Yang, "Lanthanide based whitelight-emitting hydrogel mediated by fluorescein and carbon dots with high quantum yield and multi-stimuli responsiveness," Journal of Materials Chemistry C, vol. 8, no. 10, pp. 3380-3385, 2020.

[49] S. Zhu, S. Tang, J. Zhang, and B. Yang, "Control the size and surface chemistry of graphene for the rising fluorescent materials," Chemical Communications, vol. 48, no. 38, pp. 4527-4539, 2012.

[50] F. Chen, Y. M. Wang, W. Guo, and X. B. Yin, "Color-tunable lanthanide metal-organic framework gels," Chemical Science, vol. 10, no. 6, pp. 1644-1650, 2019.

[51] K. Meng, C. Yao, Q. Ma et al., "A reversibly responsive fluorochromic hydrogel based on lanthanide-mannose complex," Advanced Science, vol. 6, no. 10, Article ID 1802112, 2019.

[52] H. I. Park and S. Y. Park, "Smart fluorescent hydrogel glucose biosensing microdroplets with dual-mode fluorescence quenching and size reduction," ACS Applied Materials \& Interfaces, vol. 10, no. 36, pp. 30172-30179, 2018.

[53] K. Ai, B. Zhang, and L. Lu, "Europium-based fluorescence nanoparticle sensor for rapid and ultrasensitive detection of an anthrax biomarker," Angewandte Chemie International Edition, vol. 48, no. 2, pp. 304-308, 2009.

[54] G. Weng, S. Thanneeru, and J. He, "Dynamic coordination of Eu-iminodiacetate to control fluorochromic response of polymer hydrogels to multistimuli," Advanced Materials, vol. 30, no. 11, Article ID 1870073, 2018.

[55] N. Mehwish, X. Dou, Y. Zhao, and C. L. Feng, "Supramolecular fluorescent hydrogelators as bio-imaging probes," Materials Horizons, vol. 6, no. 1, pp. 14-44, 2019.

[56] S. Xu, A. C. Sedgwick, S. A. Elfeky et al., "A boronic acid-based fluorescent hydrogel for monosaccharide detection," Frontiers of Chemical Science and Engineering, vol. 14, no. 1, pp. 112-116, 2020.

[57] H. Niu, X. Li, H. Li et al., "Thermosensitive, fast gelling, photoluminescent, highly flexible, and degradable hydrogels for stem cell delivery," Acta Biomaterialia, vol. 83, pp. 96-108, 2019.

[58] S. H. Park, J. Y. Seo, J. Y. Park et al., “An injectable, clickcrosslinked, cytomodulin-modified hyaluronic acid hydrogel for cartilage tissue engineering," NPG Asia Materials, vol. 11, 2019.

[59] Y. Wang, Y. Xue, J. Wang et al., "A composite hydrogel with high mechanical strength, fluorescence, and degradable behavior for bone tissue engineering," Polymers, vol. 11, no. 7, Article ID 1112, 2019.

[60] A. P. Singh, A. Biswas, A. Shukla, and P. Maiti, "Targeted therapy in chronic diseases using nanomaterial-based drug delivery vehicles," Signal Transduction and Targeted Therapy, vol. 4, no. 33, 2019.

[61] J. Li and D. J. Mooney, "Designing hydrogels for controlled drug delivery," Nature Reviews Materials, vol. 1, Article ID 16071, 2016.

[62] S. P. H. Moghaddam, J. Saikia, M. Yazdimamaghani, and H. Ghandehari, "Redox-responsive polysulfide-based biodegradable organosilica nanoparticles for delivery of bioactive agents," ACS Applied Materials \& Interfaces, vol. 9, no. 25, pp. 21133-21146, 2017.

[63] Y. Ma, H. Zhou, F. Hu, Z. Pei, Y. Xu, and Q. Shuai, "Multifunctional nanogel engineering with redox-responsive and AIEgen features for the targeted delivery of doxorubicin hydrochloride with enhanced antitumor efficiency and realtime intracellular imaging," Artificial Cells, Nanomedicine, and Biotechnology, vol. 46, no. 3, pp. S900-S910, 2018.

[64] X. Guo, D. Xu, H. Yuan et al., "A novel fluorescent nanocellulosic hydrogel based on carbon dots for efficient adsorption and sensitive sensing in heavy metals," Journal of Materials Chemistry A, vol. 7, no. 47, pp. 27081-27088, 2019.

[65] H. Ehtesabi, S. Roshani, Z. Bagheri, and M. Yaghoubi-Avini, "Carbon dots-sodium alginate hydrogel: a novel tetracycline 
fluorescent sensor and adsorber," Journal of Environmental Chemical Engineering, vol. 7, no. 5, Article ID 103419, 2019.

[66] Y. Zhang, X. Le, Y. Jian, W. Lu, J. Zhang, and T. Chen, “3D fluorescent hydrogel origami for multistage data security protection," Advanced Functional Materials, vol. 29, no. 46, Article ID 1905514, 2019.

[67] X. X. Le, W. Lu, J. He, M. J. Serpe, J. W. Zhang, and T. Chen, "Ionoprinting controlled information storage of fluorescent hydrogel for hierarchical and multi-dimensional decryption," Science China Materials, vol. 62, no. 6, pp. 831-839, 2019.

[68] D. Staneva, I. Grabchev, and P. Bosch, "Fluorescent hydrogeltextile composite material synthesized by photopolymerization," International Journal of Polymeric Materials and Polymeric Biomaterials, vol. 64, no. 16, pp. 838-847, 2015.

[69] B. Li, Y. Zhang, B. Yan et al., "A self-healing supramolecular hydrogel with temperature-responsive fluorescence based on an AIE luminogen," Rsc Advances, vol. 10, no. 12, pp. 7118-7124, 2020.

[70] W. Yang, X. Wu, F. Liu, Y. Dou, Z. Hu, and W. Hao, "A fluorescent, self-healing and $\mathrm{pH}$ sensitive hydrogel rapidly fabricated from HPAMAM and oxidized alginate with injectability," RSC Advances, vol. 6, no. 41, pp. 34254-34260, 2016.

[71] C. Qian, R. Wang, M. Li et al., "Facile preparation of selfassembled black phosphorus-based composite LB films as new chemical gas sensors," Colloids and Surfaces A: Physicochemical and Engineering Aspects, vol. 608, Article ID 125616, 2021.

[72] J. Bai, R. Wang, M. Ju, J. Zhou, L. Zhang, and T. Jiao, “Facile preparation and high performance of wearable strain sensors based on ionically cross-linked composite hydrogels," Science China Materials, 2020.

[73] M. Cao, Y. Shen, Z. Yan et al., "Extraction-like removal of organic dyes from polluted water by the graphene oxide/ PNIPAM composite system," Chemical Engineering Journal, vol. 405, Article ID 126647, 2021.

[74] L. Ge, M. Zhang, R. Wang et al., "Fabrication of CS/GA/RGO/ Pd composite hydrogels for highly efficient catalytic reduction of organic pollutants," RSC Advances, vol. 10, no. 26, pp. 15091-15097, 2020.

[75] R. Su, H. Wang, Y. Sun, and P. Guo, "Structural regulation of porous $\mathrm{MnO}_{2}$ nanosheets and their electrocapacitive behavior in aqueous electrolytes," Colloids and Surfaces A: Physicochemical and Engineering Aspects, vol. 609, Article ID 125579, 2021.

[76] C. Qian, J. Yin, J. Zhao et al., "Facile preparation and highly efficient photodegradation performances of self-assembled artemia eggshell-ZnO nanocomposites for wastewater treatment," Colloids and Surfaces A: Physicochemical and Engineering Aspects, vol. 12, 2020.

[77] M. Liu, G. Ouyang, D. Niu, and Y. Sang, "Supramolecular gelatons: towards the design of molecular gels," Organic Chemistry Frontiers, vol. 5, no. 19, pp. 2885-2900, 2018. 